\title{
Prediction of the Transmission Loss in a Flexible Chamber
}

\author{
A. J. Torregrosa ${ }^{\mathrm{a}}$, A. Gila ${ }^{\mathrm{a}}$, L. M. García-Cuevas ${ }^{\mathrm{a}}$, P. Quintero ${ }^{\mathrm{a}, *}$, F. D. Denia ${ }^{\mathrm{b}}$ \\ ${ }^{a}$ CMT-Motores Térmicos, Universitat Politècnica de València, Camino de Vera s/n, 46022 Valencia, Spain. \\ ${ }^{b}$ Centro de Investigación en Ingeniería Mecánica, Universitat Politècnica de València, Camino de Vera s/n, 46022 Valencia, Spain.
}

\begin{abstract}
Acoustic components have been extensively studied supposing perfectly rigid behavior. Although some works have been performed for the radiated sound in the case of a flexible element, an important lack of information exists concerning transmission loss analysis. The current investigation proposes the study for a generic flexible expansion chamber. The analysis has been performed using two different methods: a resolution in the time domain, using a Finite Volume discretization for the fluid domain and a Finite Element discretization for the solid domain, and an approach in the frequency domain, using a Finite Element discretization for both fluid and solid. After studying the rigid case in order to tune up the simulation, the study of the flexible case shows a good agreement among both methods. The comparison of rigid and flexible expansion chambers shows the importance of accounting for these phenomena when the frequency content of the acoustic signal excites the natural modes of the structure.
\end{abstract}

Keywords: silencer, muffler, expansion chamber, finite element method, finite volume method, flexibility, deformation, acoustics, vibroacoustics, fluid structure interaction, 2 way FSI

\section{Introduction}

Reduction of noise emissions is currently an important area of interest because of its practical importance. A document of the World Health Organization for the European Union [1] showed that near $40 \%$ of citizens of the EU experience road noise of about $55 \mathrm{~dB}(\mathrm{~A})$. A $30 \%$ of European population experiences road noise over $55 \mathrm{~dB}(\mathrm{~A})$ during night.

Recent regulations focus their application on an effective reduction of noise [2]. In order to comply with regulation without jeopardizing engine performance, various noise control methods have been developed. These kind of control methods can be categorized as passive or active systems [3].

Active noise techniques allow obtaining a very high reduction of observer perceived noise. However, this kind of control is associated with some issues of cost and reliability. Currently, their application on the transport industries is not approachable. For instance, Linus et al. [4] demonstrated that the performance of active-noise cancelling headphones is dependent on the noise environment. Under some circumstances, they showed how its performance could be unacceptable. In the automotive field it is necessary for a control mechanism to be useful on the whole range of operation making the use of this kind of devices currently out of scope.

Due to these limitations, passive noise control is nowadays the principal engineering solution. A general categorization of passive elements can be split as dissipative or reflective. Dissipative noise control allows a high noise reduction. For example, Hwang et al. [5] showed how using dissipative viscoelastic materials allows an effective noise control. However, porous absorption materials lose acoustic performance for low frequencies. When the frequency is low, the thickness of a porous absorber is less than one quarter of the acoustic wavelength and absorption becomes inappreciable [6].

For reactive noise abatement techniques, part of the sound wave is reflected towards the source, or back and forth among the elements. Some examples of these elements are: expansion chambers, Helmholtz resonators, Herschel Quinke tubes, etc. These elements allow dealing with low frequency noise [7].

${ }^{*}$ Corresponding author. Tel.: +34 963877650 , fax: +34963877659.

Email address: pedqui ig@mot.upv.es (P. Quintero) 
Traditionally, reactive elements have been extensively considered as infinitely rigid, i.e. wall displacement and/or velocity is supposed to be negligible from the fluid behavior point of view. This is effectively true in most of the current applications in the automotive industry. However, very low density-low rigidity materials are becoming of interest. As an example it could be useful to refer to the works of Aydemir and Ebrin [7], Nunes et al. [8] and Hu et al. [9].

Some flexible components have been proposed in the field of passive sound attenuation. For example, Huang [10] analyzed a drumlike silencer, which is a strongly coupled fluid-structure component. It was shown how the coupling of the structural eigenfrequencies with the flow leads to a kind of "storage" of kinetic energy, yielding to an increase of the Transmission Loss (TL) through the main duct.

Some approximations have been made in this context for some academic problems. For example, Fan et al. [11] used numerical methods to compute the fluid-structure interaction of a flexible panel immersed in a fluid flow; Lawrie [12] , Ramamoorthy et al. [13] and Ko [14] analytically approached the behavior of canonical cases. Gautier et al. [15] performed measurements on the vibroacoustic phenomena appearing on a flexible rubber tube. Practical geometries have been studied less extensively, but some references could be cited: Venkatesham et al. [16] developed an analytical prediction for the radiated sound from a rectangular flexible expansion chamber; Wang et al. [17] studied the radiated sound from a rectangular cavity through an elastic panel.

Nevertheless, there exists an important lack of information about the influence of structural phenomena on the transmission properties, being of vital importance the quantification of the transmission loss and/or transfer matrix coefficients. For example, Munjal and Thawani [18] analytically showed how transmission loss should increase when decreasing the rigidity of the wall material. Cummings [19] developed a theoretical model describing acoustic attenuation in a flexible walled duct passing through a reverberant space.

Due to the mentioned lack of information, in the present work numerical modelling has been applied to a typical circular-section expansion chamber. This geometry was characterized via the component acoustic transfer matrix [6] for the rigid case and for various flexible materials.

The expansion chamber studied during this work consists on a cylindrical geometry with only one flexible wall. The reason of selecting this kind of geometry is mainly due to its simplicity and, thus, to the possibility of inferring general behaviors for more complex geometries. As it will be shown later, due to the inherently stiffness of this geometry, the material must have a very low Young modulus in order to show fully coupled fluid structure interactions under acoustic loads. The main aim of this work is to prove how, when structural modes are excited, it is necessary to consider couplings and to provide appropriate methodologies to predict the behavior of the acoustic element under these circumstances.

The present work is structured as follows: first, in section 2 the theoretical background is analyzed, both for the fluid and the solid domain; then, section 3 provides a complete description of the domain studied, in order to ensure that present simulations are completely reproducible, while section 4 provides a complete description of the modelling performed (meshes, fluid, etc.). Then, the results obtained for the rigid and the flexible expansion chamber are described and analyzed in sections 5 and 6, respectively, and finally section 7 summarizes the most important conclusions deduced from the present study.

\section{Theoretical Background}

For the solution of this problem, two different zones must be considered: A fluid zone, where sound waves propagate, and a solid zone, which experiences deformations and accelerations as a response to flow characteristics. In order to study the transmission characteristics with flexible walls, a full coupled case must be studied, i.e. as a consequence of wave propagation the solid walls will experience an unsteady deformation which in turn will also have an effect on the unsteady flow field [20]. In this section, the governing equations for each region are summarized.

\subsection{Solid continua equations}

\subsubsection{General equations}

The equations governing fluid flow and structural solid displacement are essentially the same as those presented in the work of Zienkiewicz et al. [21]. The unsteady governing equations for the solid media can be written in vector form as follows: 


$$
\rho \frac{\partial^{2} \vec{w}}{\partial^{2} t}=\nabla \cdot \sigma-\vec{b}
$$

Here, $\vec{w}$ is the vector representing the displacement field of the solid body, measured from an inertial reference frame; $\vec{b}$ represents the volume forces (as gravity or dissipation); $\rho$ is the material density and $\sigma$ is the Cauchy stress tensor [22], which complies with:

$$
\sigma=\sigma^{T}
$$

The closure of the solid displacement equation can be obtained by means of the so-called strain-stress relationships. Let the solid strains be defined as:

$$
\varepsilon_{i j}=\frac{1}{2}\left(\frac{\partial w_{i}}{\partial x_{j}}+\frac{\partial w_{j}}{\partial x_{i}}\right)
$$

Then, the strain-stress equation allows to set a relationship between strain and stress for a given material. For instance, in the case of a linear-homogeneous solid material:

$$
\sigma_{i j}=\lambda \delta_{i j} \varepsilon_{i j}+2 \mu \varepsilon_{i j}
$$

where $\delta_{i j}$ is the Kronecker delta and $\lambda$ and $\mu$ are the first and second Lamé parameters, respectively. They are usually expressed as a function of the Young modulus, $E$, and Poisson ratio, $v$, as follows:

$$
\lambda=\frac{E v}{(1+v)(1-2 v)} \quad \mu=\frac{E}{2(1+v)}
$$

Once the equations are closed and suitable boundary and initial conditions are prescribed, the resulting linear problem can be efficiently solved by means of the Finite Element Method (FEM) [21].

\subsubsection{Free vibration equations}

The set of discrete equations describing the solid structural domain can be obtained by means of the FEM [21] and is written simply as:

$$
[M]\{\ddot{w}\}+[C]\{\dot{w}\}+[K]\{w\}=\left\{f^{e x t}\right\}
$$

where $[M]$ is the mass matrix, $[C]$ is the damping matrix, $[K]$ is the stiffness matrix, $\left\{f^{e x t}\right\}$ is the vector of nodal forces and $\{w\}$ is the vector of nodal displacements.

The free vibration problem consists in solving the following problem (with $\left.\{w\}=\{W\} e^{j \omega t}\right)$ :

$$
-\omega^{2}[M]\{W\}+j \omega[C]\{W\}+[K]\{W\}=0
$$

The values of $\omega$ that satisfy the previous equation are the so-called eigenfrequencies. Any excitation with this frequency can lead to very high values in the displacement. As it will be seen later, eigenfrequencies must be computed because of their great importance when analyzing fluid-structure interaction (FSI) harmonic acoustic problems.

\subsection{Fluid motion equations}

\subsubsection{General equations}

In this study the propagation of a velocity/pressure pulse through a fluid domain has been modelled. The most straight-forward derivation of the equations governing this system is based on applying the equation of state in conjunction with the mass, momentum and energy equations [23], which are given by the following expressions:

$$
\left\{\begin{array}{c}
p=\rho R T \\
\frac{\partial \rho}{\partial t}+\nabla \cdot(\rho \vec{u})=0 \\
\rho\left(\frac{\partial \vec{u}}{\partial t}+\vec{u} \cdot \nabla \vec{u}\right)=\nabla \cdot \tau+\rho \overrightarrow{f_{m}} \\
\rho\left(\frac{\partial h}{\partial t}+\vec{u} \cdot \nabla h\right)=\frac{\partial p}{\partial t}+\vec{u} \cdot \nabla p+\nabla(k \nabla T)+\phi_{v}+Q \\
\mathrm{~d} h=c_{p} \mathrm{~d} T
\end{array}\right.
$$




\subsubsection{Euler Equations}

For the propagation of low to moderate wave amplitudes the flow can be considered to behave as non-viscous $(\mu \approx 0)$ and heat-transfer dissipative effects can also be neglected $(k \approx 0)$. Also body forces and heat generation will be neglected (see [24] and [25]).

After considering those assumptions, the Euler equations are obtained. These are given by:

$$
\left\{\begin{array}{c}
p=\rho R T \\
\frac{\partial \rho}{\partial t}+\nabla \cdot(\rho \vec{u})=0 \\
\frac{\partial \vec{u}}{\partial t}+\vec{u} \nabla \vec{u}=-\frac{\nabla p}{\rho} \\
\rho\left(\frac{\partial h}{\partial t}+\vec{u} \cdot \nabla h\right)=\frac{\partial p}{\partial t}+\vec{u} \cdot \nabla p \\
\mathrm{~d} h=c_{p} \mathrm{~d} T
\end{array}\right.
$$

For the FSI-coupled case, a morphing mesh scheme is adopted and the wall-velocity inviscid boundary condition can be expressed simply by:

$$
\left(\vec{u}_{\text {fluid }} \cdot \vec{n}\right)_{\text {wall }}=\vec{u}_{\text {wall }} \cdot \vec{n}
$$

The previous equation simply states that fluid and solid must have the same normal velocity components at the interface.

\subsubsection{Wave equation}

In the context of wave motion, it is usual to assume that the flow characteristics can be determined as an unperturbed component and a perturbation as follows:

$$
\begin{aligned}
\rho_{T}(\vec{x}, t) & =\rho_{0}(\vec{x}, t)+\rho(\vec{x}, t) \\
p_{T}(\vec{x}, t) & =p_{0}(\vec{x}, t)+p(\vec{x}, t) \\
\vec{u}_{T}(\vec{x}, t) & =\vec{u}_{0}(\vec{x}, t)+\vec{u}(\vec{x}, t)
\end{aligned}
$$

where $\frac{\rho}{\rho_{0}} \ll 1 ; \frac{p}{p_{0}} \ll 1$ and $\frac{|\vec{u}|}{|\vec{u}|_{0}} \ll 1$. Under those circumstances the Euler equations can be linearized. Also, it is possible to consider the flow to be isentropic, allowing one to define a relation between pressure and density derivatives, as:

$$
\left(\frac{\partial p}{\partial \rho}\right)_{s}=\left(\frac{\partial p}{\partial \rho}\right)=a_{0}^{2}
$$

Where $a_{0}$ is the unperturbed sound velocity in the flow. Finally, for the case of no mean flow the formulation can be further simplified, and a single equation for the perturbation (acoustic) pressure is obtained:

$$
\nabla^{2} p-\frac{1}{a_{0}^{2}} \frac{\partial^{2} p}{\partial^{2} t}=0
$$

allowing also to obtain the acoustic velocity, which is related to pressure derivatives as follows:

$$
\frac{\partial \vec{u}}{\partial t}=-\frac{1}{\rho_{0}} \nabla p
$$

\subsubsection{Helmholtz equation}

Assuming harmonic dependence with time of the acoustics variables (i.e. $p=P e^{j \omega t}$ and $u=U e^{j \omega t}$ ), it is possible to obtain the Helmholtz equation, which can be written as [6]:

$$
\nabla^{2} P+\frac{\omega^{2}}{a_{0}^{2}} P=0 \rightarrow \nabla^{2} P+k_{0}^{2} P=0
$$

and the corresponding relationship between pressure and velocity: 


$$
\vec{U}=\frac{j}{\omega \rho_{0}} \nabla P
$$

Eq.(15) can be numerically solved by means of a linear FEM [26]. For the solution of the domain sketched in section 3, the commercial package COMSOL was used [27].

\section{Problem Description}

The main aim of this study is to characterize a flexible-wall expansion chamber using time-domain CFD methods, and to compare the results with a frequency domain FEM solution. A similar problem was already studied by Broatch et al. [28] for the case of an mufflers with rigid walls.

Due to the geometrical characteristics of the fluid domain, the plane wave condition, $\frac{f R}{a_{0}} \ll 0.29$ is sufficiently adequate for the range of frequencies of interest, i.e. $0<\frac{f R}{a_{0}}<0.13$. Nevertheless, in order to be able to predict possible non-planar wave effects while avoiding the use of a too large domain, only one quarter of a tube was simulated, assuming periodicity in the angular coordinate.

As it will be checked later, the results for this simple case can be considered as practically axisymmetric. Thus, the simulation of a quarter of a pipe is considered to be representative enough for representing the whole $3 \mathrm{D}$ fluid-structure interactions.

Fig. 1 shows an sketch of the geometry to be studied and characterized. The radius of the expansion chamber is $R=0.03 \mathrm{~m}$. The solid wall is supposed to be clamped at the beginning and ending of the expansion chamber. Table 1 shows the other dimensions.

In the simulation, the inlet is fed by a known velocity profile. The outlet section of the tube is extruded a distance $L_{d i s s}=10 R$ and at its end a dissipative boundary condition is applied in order to simulate an anechoic termination. The time evolution of the inlet velocity profile is given by:

$$
\frac{v(t)}{v_{\text {max }}}=\left\{\begin{array}{rr}
\frac{1}{2}\left(1-\cos \left(\frac{2 \pi t}{T_{\text {impulse }}}\right)\right) & t \leq T_{\text {impulse }} \\
0 & t>T_{\text {impulse }}
\end{array}\right.
$$

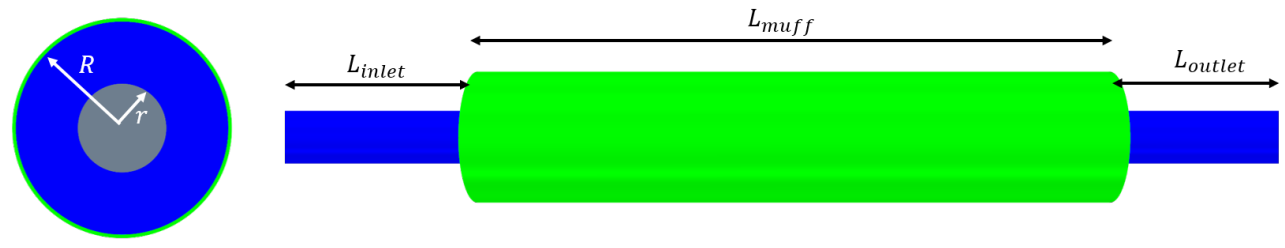

Figure 1: Sketch of the geometry

Table 1: Geometry dimensions

\begin{tabular}{|c|c|c|}
\hline \multicolumn{2}{|c|}{ Variable } & Value \\
\hline Chamber radius & $R$ & $0.03 \mathrm{~m}$ \\
\hline Inlet/Outlet radius & $r$ & $R / 2.4$ \\
\hline Inlet length & $L_{\text {inlet }}$ & $10 R / 3$ \\
\hline Outlet length & $L_{\text {outlet }}$ & $10 R / 3$ \\
\hline Chamber length & $L_{\text {muff }}$ & $10 R$ \\
\hline Dissipation length & $L_{\text {diss }}$ & $10 R$ \\
\hline
\end{tabular}


Due to the non-linearity of the convective term and the isentropic state relationship in Euler equations, high values of the velocity or pressure at boundary conditions could lead to a non-linear response. For the current case, simulations were performed for excitations up to $10 \mathrm{~m} / \mathrm{s}$, where this influence began to be slightly noted at high frequencies. Thus, a low velocity excitation of $v_{\max }=0.01 \mathrm{~m} / \mathrm{s}$ was chosen. This value is high enough to not provide numerical inaccuracies and ensures that the response can be considered to be totally linear for the whole spectra.

The non-dimensional pulse duration is given by $\frac{T a_{0}}{L_{m u f f}}=0.3473$ in such a way that the signal frequency content is high enough to resolve up to the desired frequency.

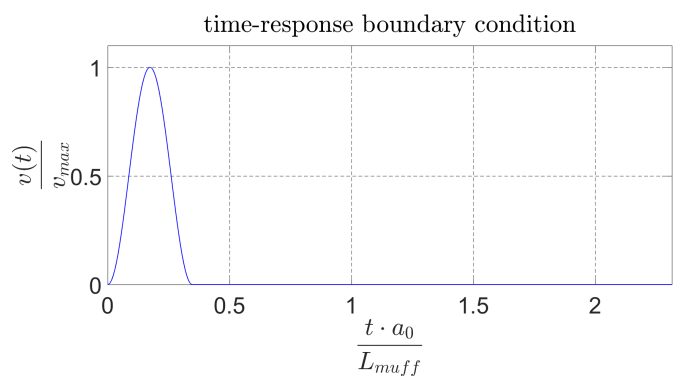

(a)

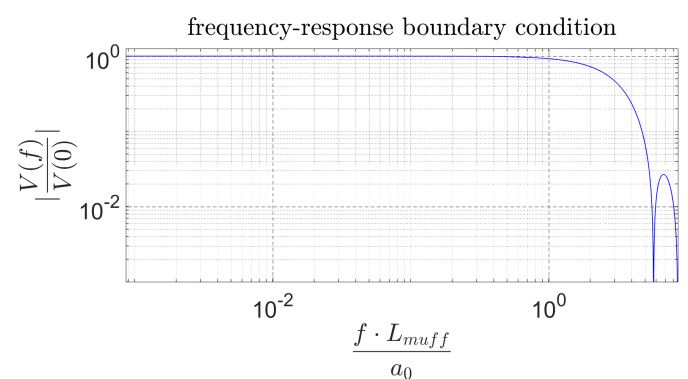

(b)

Figure 2: Time and frequency response of the inlet velocity profile

Figs. $2 \mathrm{a}$ and $2 \mathrm{~b}$ show the time and frequency evolution of the inlet velocity profile. It should be noticed that the cut-off frequency at the boundary condition $\frac{f_{\text {cut }} L_{\text {muff }}}{a_{0}} \approx 4.75$ is sufficiently high for the current requirements.

During next sections, first, acoustic transmission through the rigid expansion chamber are being calculated by means of a time-domain CFD simulation, by a FEM wave equation solution and by an analytical calculation. Furthermore, additional numerical calculations were performed for the case of a flexible expansion chamber, with wall thickness $\frac{\delta}{R}=\frac{1}{15}$ and different Young modulus, $E$, and solid density, $\rho$.

Liu et al. [29] demonstrated for a similar domain that the acoustic properties of the flexible muffler are determined by the non-dimensional parameters listed below:

$$
\begin{array}{ccc}
L_{\text {muff }}^{*}=\frac{L_{\text {muff }}}{R} & t^{*}=\frac{a_{0} \cdot t}{L_{m u f f}} \quad f^{*}=\frac{f \cdot L_{\text {muff }}}{a_{0}} \\
p^{*}=\frac{p}{\rho_{0} a_{0}^{2}} & D^{*}=\frac{E \delta^{3}}{12\left(1-v^{2}\right) R^{3} \rho_{0} a_{0}^{2}} & m^{*}=\frac{\rho_{s} \delta}{\rho_{0} R}
\end{array}
$$

During the development of this work, unless otherwise specified, a high mass pipe is assumed. Thus the mass parameter is assumed to be constant and equal to $m^{*}=56.6$. This allows for the direct examination of the dependence with stiffness. 


\section{Numerical and analytical methods}

The case of a simple non-deformable expansion chamber has been extensively studied in the literature: Broatch et al. [28] studied expansion and reversing chamber mufflers by using a time-domain CFD method; Barbieri and Barbieri [30] applied the Finite Element Method to study a similar problem. A number of references can also be found which address the problem analytically (see [6]).

References [28] and [30] allow to conclude that, for the case of linear duct acoustics when the presence of a mean flow is not important, frequency domain FEM provides quite similar results while its computational cost is significantly lower as the time domain does not need to be resolved, being possible to model only the required frequencies.

For CFD calculations the whole "residence-time" of the wave inside the domain of interest must be solved in order to obtain an admissible frequency response. Thus, it is intended here to provide a check of the validity of the current methods for later comparison in the flexible case.

\subsection{Analytical formulation}

For the analytical plane wave approach of the model, it can be split into five parts, as shown in Fig. 3:

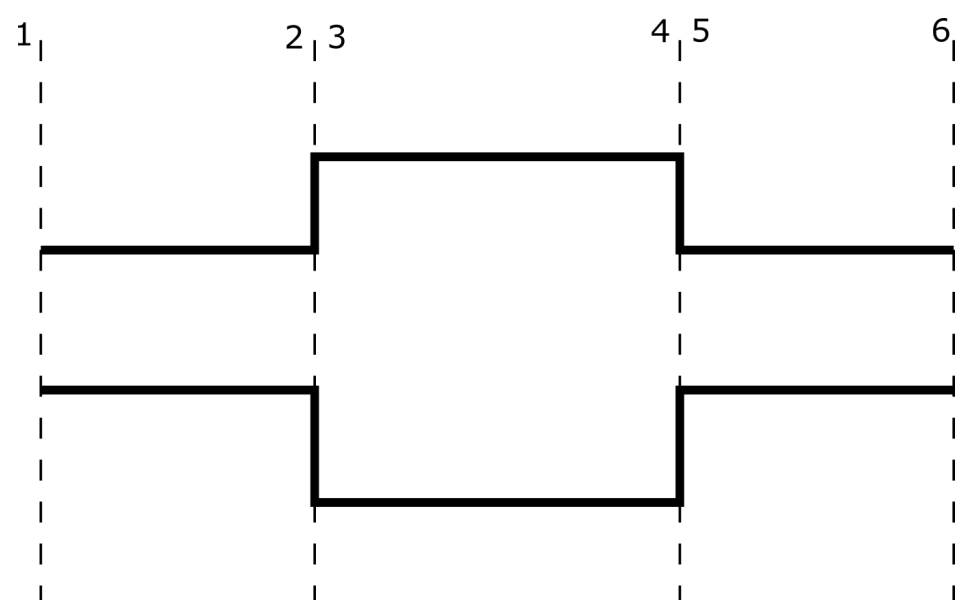

Figure 3: Simplified sketch of the geometry

Analytically, each component of the expansion chamber elements can be modelled as an acoustic transfer matrix, which relates the acoustic velocity and pressure at the inlet section with those at the outlet. For an arbitrary acoustic element it can be stated [6]:

$$
\left\{\begin{array}{l}
p_{\text {in }} \\
u_{\text {in }}
\end{array}\right\}=[A]\left\{\begin{array}{l}
p_{\text {out }} \\
u_{\text {out }}
\end{array}\right\}=\left[\begin{array}{ll}
A_{11} & A_{12} \\
A_{21} & A_{22}
\end{array}\right]\left\{\begin{array}{l}
p_{\text {out }} \\
u_{\text {out }}
\end{array}\right\}
$$

It follows that, for a linear element consisting of $n$ subelements whose transfer matrices are known one can write [6]:

$$
[A]=\left[A_{1}\right]\left[A_{2}\right] \cdots\left[A_{n}\right]
$$

where $\left[A_{i}\right]$ is the transfer matrix of the $i$ sub-element. The transfer matrix of each one has been extensively analyzed in bibliography [6].

Once the acoustic matrix of a system is known it is possible to predict the its acoustical behavior under any harmonic excitation. On the other hand, it is also possible to predict an important acoustic characteristic of the system as follows:

On the other hand, it is also possible to predict the transmission loss (TL) of the system. This parameter represents the quantity of sound power which leaves the acoustic element, related to the incident power provided that the outlet is anechoic [6]. It is usually expressed in $\mathrm{dB}$, and can be deduced from the elements of the transfer matrix: 


$$
\begin{array}{cc}
T L= & 10 \log _{10}\left(\frac{W_{\text {out }}}{W_{\text {in }}}\right) \\
T L=20 \log _{10}\left[\sqrt{\frac{S_{\text {out }}}{S_{\text {in }}}} \frac{\left|A_{11}+A_{12} \cdot \frac{S_{\text {in }}}{\rho_{0} a_{0} S_{\text {out }}}+A_{21} \rho_{0} a_{0} \frac{S_{\text {in }}}{S_{\text {out }}}+A_{22}\left(\frac{S_{\text {in }}}{S_{\text {out }}}\right)^{2}\right|}{2}\right]
\end{array}
$$

Where $S_{\text {out }}$ represents the outlet section of the element and $S_{\text {in }}$ is the inlet section. When $S_{\text {out }}=S_{\text {in }}$ it can be stated:

$$
T L=20 \log _{10}\left(\frac{\left|p_{\text {out }}\right|^{+}}{\left|p_{\text {in }}\right|^{+}}\right)
$$

$\left|p_{\text {out }}\right|^{+}$and $\left|p_{\text {in }}\right|^{+}$being the progressive pressure wave component at outlet and inlet, respectively.

\subsubsection{Numerical calculation of the transfer matrix}

As it was shown in the previous section, the transfer matrix is composed by 4 coefficients. Thus, in order to determine it from a numerical computation two cases have to be calculated. Once the frequency content of the acoustic pressure and velocity is known, the value of the matrix components, for a particular frequency $f$ can be evaluated as follows:

$$
\left[\begin{array}{l}
A_{11}(f) \\
A_{12}(f) \\
A_{21}(f) \\
A_{22}(f)
\end{array}\right]=\left[\begin{array}{cccc}
\left(P_{\text {out }}(f)\right)_{\text {Case } A} & \left(V_{\text {out }}(f)\right)_{\text {Case } A} & 0 & 0 \\
0 & 0 & \left(P_{\text {out }}(f)\right)_{\text {Case A }} & \left(V_{\text {out }}(f)\right)_{\text {Case A }} \\
\left(P_{\text {out }}(f)\right)_{\text {Case B }} & \left(V_{\text {out }}(f)\right)_{\text {Case B }} & 0 & 0 \\
0 & 0 & \left(P_{\text {out }}(f)\right)_{\text {Case B }} & \left(V_{\text {out }}(f)\right)_{\text {Case B B }}
\end{array}\right]^{-1}\left[\begin{array}{c}
\left.\left(P_{\text {in }}(f)\right)_{\text {Case } A}\right) \\
\left.\left(V_{\text {in }}(f)\right)_{\text {Case } A}\right) \\
\left.\left(P_{\text {in }}(f)\right)_{\text {Case B }}\right) \\
\left.\left(V_{\text {in }}(f)\right)_{\text {Case B }}\right)
\end{array}\right]
$$

The previous equation can be resolved only when the boundary conditions of cases A and B are linearly independent in the frequency domain.

\subsection{Time domain CFD Model}

In section 2.2.2 the Euler equations were introduced. In order to solve them, a general-purpose commercial software, STARCCM+, has been used.

The fluid considered was air. For the working conditions (i.e. $p_{0}=101325 \mathrm{~Pa}$ and $T_{0}=300 \mathrm{~K}$ ), this fluid behaves as a perfect gas characterized by a gas constant $R=287.02 \mathrm{~J} \mathrm{~kg}^{-1} \mathrm{~K}^{-1}$, an adiabatic index $\gamma=1.4$, an unperturbed sound velocity $a_{0}=347.28 \mathrm{~m} \mathrm{~s}^{-1}$ and unperturbed density $\rho_{0}=1.177 \mathrm{~kg} \mathrm{~m}^{-3}$.

The selection of a mesh size of the fluid volume-domain must be a compromise between the maximum desired frequency resolved (in this case $\frac{f L_{m u f f}}{a_{0}}=1.30$ ) and a correct discretization of the circular pipe-domain. In this case this size was taken to be $\frac{R}{\Delta x}=24$.

About the selection of the time-step, as wave propagation is the phenomenon of interest, a low acoustic Courant number $(C F L)$ must be set, based on the wave-speed velocity. In this case, it was taken as $C F L=\frac{a_{0} \Delta t}{\Delta x}<1 \rightarrow \frac{a_{0} \Delta t}{\Delta x}=$ $1.2 \cdot 10^{-3}$.

A visualization of the fluid mesh is shown in Fig. 4(a). The coupled solid problem (1) was also solved by using the commercial software STARCCM+. Therefore, a FEM mesh was created to model the solid domain.

Limitations of the time domain solver used during this work lead to the use of second order 3D solid elements in order to model the solid domain, making the solution of the structural problem more CPU consuming than if shell elements would have been used. Nevertheless, as the number of elements of the flexible wall is much lower that for the fluid domain, this element selection will not become a bottleneck. Thus, the solution of a thin walled solid with 3D elements requires at least three elements across the thickness [31]. Therefore, the surface was modeled by using second order hexahedral elements of side $\frac{R}{\Delta x}=10$. Fig. 4(b) shows a representation of the solid mesh. It should be noted that, as already mentioned, the use of shell elements could improve the computational effort with negligible effects in the accuracy of the results. 


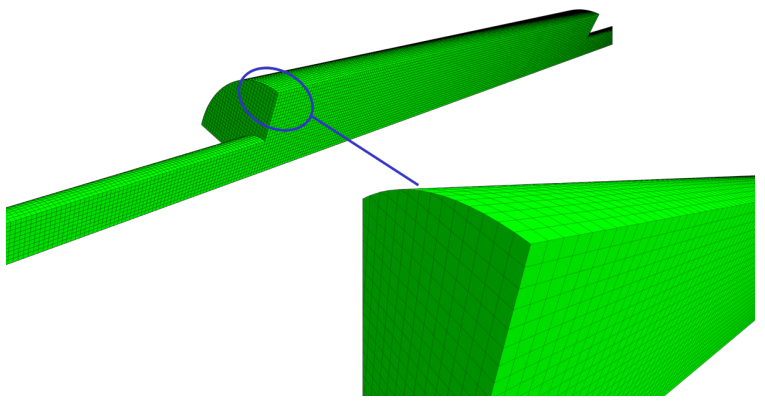

(a) Finite Volume mesh

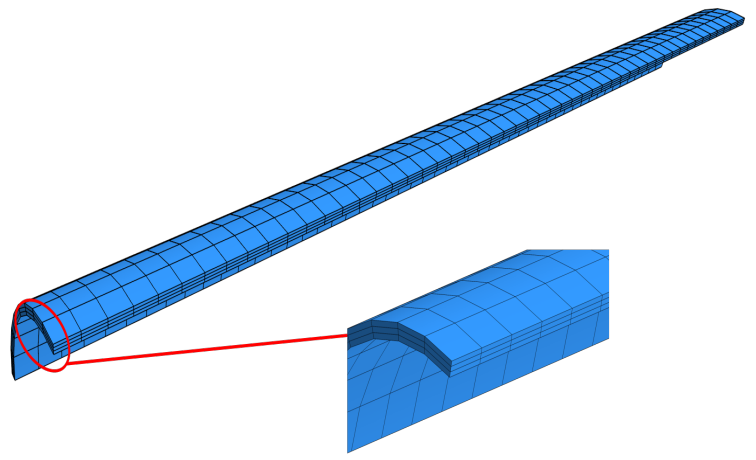

(b) Finite Element mesh

Figure 4: STARCCM+ mesh visualisation

\subsubsection{Description of cases for the numerical determination of the transfer matrix coefficients using CFD}

As previously shown, in order to get the values of the transfer matrix coefficients, resolution of variables for two different cases must be performed. These cases are hereinafter referred to as "Case A" and "Case B". Description of each case is given below.

Case A. For the first case the inlet velocity history is assumed to be known as shown in Equation (17). The outlet of the pipe is set to be anechoic, so that no pressure reflections are found in this boundary.

Case B. For the second case the inlet velocity history is supposed to be known as shown in Equation (17) until the velocity pulse is introduced into the domain. Once the pulse is introduced, this boundary condition is set to be anechoic, so that not pressure reflections are found in the inlet.

The outlet boundary condition was set to be as a rigid wall, so at this section the velocity history is supposed to be known and equal to $v_{\text {out }}(t)=0$.

\subsection{Frequency domain FEM Model}

In section 2.2.4, the Helmholtz Equation was introduced. In order to solve it, the general-purpose commercial software COMSOL was used. Compromise between geometry and frequency mesh size requirements can be achieved by using a uniform mesh size which could be excessive from the frequency point of view, but adapts well to the geometry [32].

Compared with the CFD model, and due to the linearity of the solved equation, the mesh requirements are significantly lower for the FEM case. Thus, for this case mesh size was taken to be $\frac{R}{\Delta x}=6$ for the whole domain, using quadratic elements. Fig. 5 shows a representation of the FEM mesh. As a disadvantage it can be cited that only linear effects are computed. 


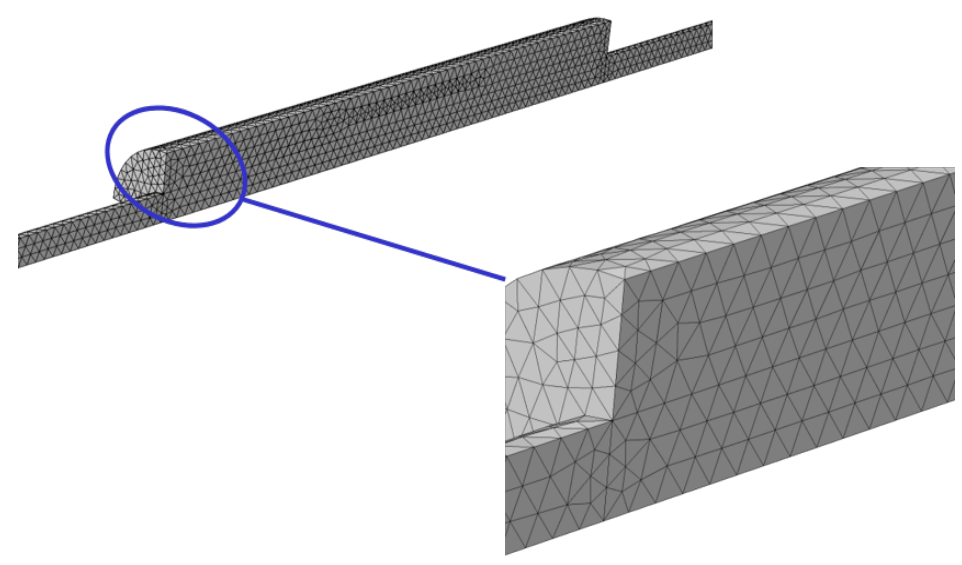

Figure 5: COMSOL Fluid domain mesh

The solid domain was modelled using a zero-thickness shell approximation, which allows lower CPU time consumption with no significant loss of accuracy, as it was mentioned before. This kind of model allows to account for the whole thickness in the case of thin walls by only using one surface element.

\subsubsection{Description of cases for the numerical determination of the transfer matrix coefficients using FEM}

As previously shown, in order to get the value of the transfer matrix coefficients, solution of variables for two different cases must be performed. Again, these cases are hereinafter referred to as "Case A" and "Case B". Description of each case is given below.

Case A. For the first case the inlet pressure is supposed to be harmonic, evolving in accordance with:

$$
p_{\text {in }}(t)=P_{\text {in }} e^{j \omega t} \quad P_{\text {in }}=1 \mathrm{~Pa}
$$

The outlet face is assumed to be anechoic. Thus, the acoustic impedance is known, with value:

$$
Z_{\text {out }}=Z_{0}=\rho_{0} a_{0}
$$

It should be recalled that, due to the linearity of the Helmholtz equation, the selection of the inlet pressure is completely arbitrary, because it will have no influence on the quantification of the acoustic element features.

Case B. Case B uses the same inlet conditions as does Case A. The outlet section is again assumed to behave as a rigid wall. Thus, the acoustic normal velocity is known, with value:

$$
v_{\text {out }}=0
$$

\section{Results for the rigid expansion chamber}

\subsection{CFD time domain results}

When the maximum inlet velocity is set to $v_{\max }=0.01 \mathrm{~ms}^{-1}$, the maximum value of the inlet pressure pulse can be found to be $p_{\max }=4.081 \mathrm{~Pa}$. For a correct time domain CFD simulation, the calculation must be run until the whole inlet pulse leaves the domain through the non-reflecting boundary conditions. For the present case all the simulations were performed until a final time of $\frac{t a_{0}}{L_{m u f f}}=100$. This value ensures that all the energy supplied at the inlet leaves the domain. 
In order to obtain the transmission characteristics of the system it is necessary to perform a computation of both pressure and velocity at the inlet and the outlet for each case studied (Case A and Case B). These values are shown in Fig. 6.
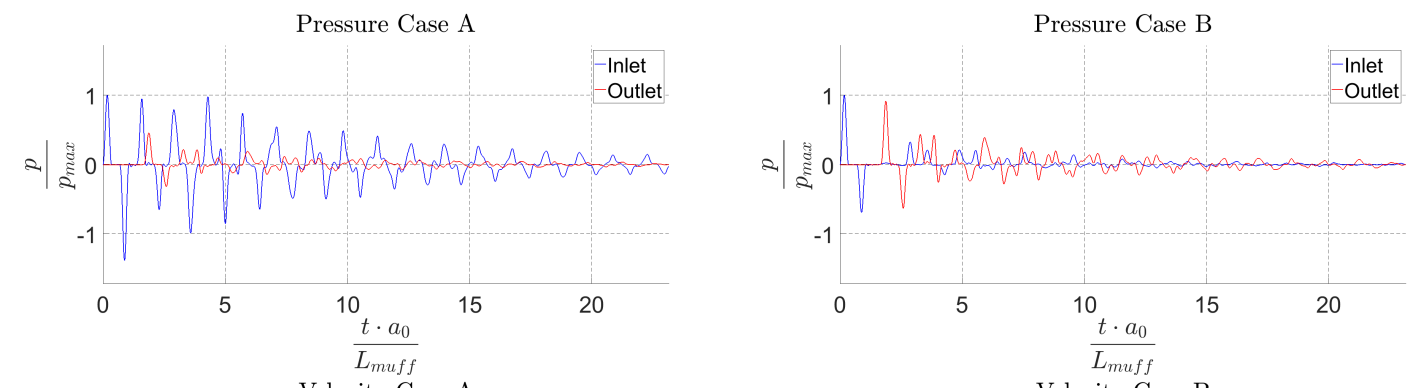

Velocity Case A
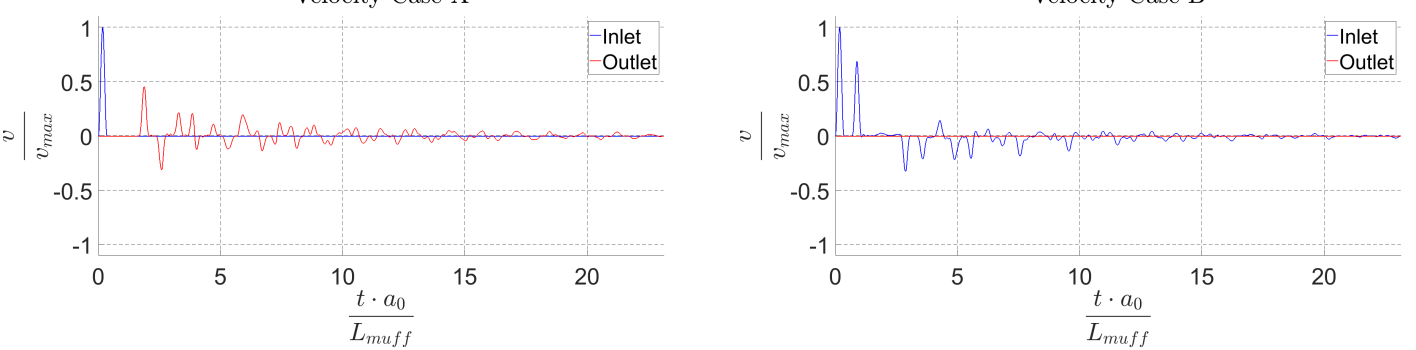

Figure 6: Time evolution of the pressure and velocity at domain boundaries

In order to obtain the frequency characteristics of the expansion chamber, a Discrete Fourier Transform must be applied to the boundary data. It is thus supposed that a function $x(t)$ can be approximated by a Fourier Series, as indicated by the following expression:

$$
X_{k}=\sum_{n=0}^{N-1} x_{n} e^{-j \frac{2 \cdot \pi \cdot k \cdot n}{N}}
$$

where $x_{n}$ is the pressure at sampling time $n, N$ is the number of samples and $X_{k}$ is the frequency response for the $k^{\text {th }}$ frequency. Applying this concept to the computed time signals the frequency response for each of the cases are obtained. These are shown in Fig. 7. Sampling time is taken to be $T_{s}=1.2 \cdot 10^{-3} \Delta x / a_{0}$ : 

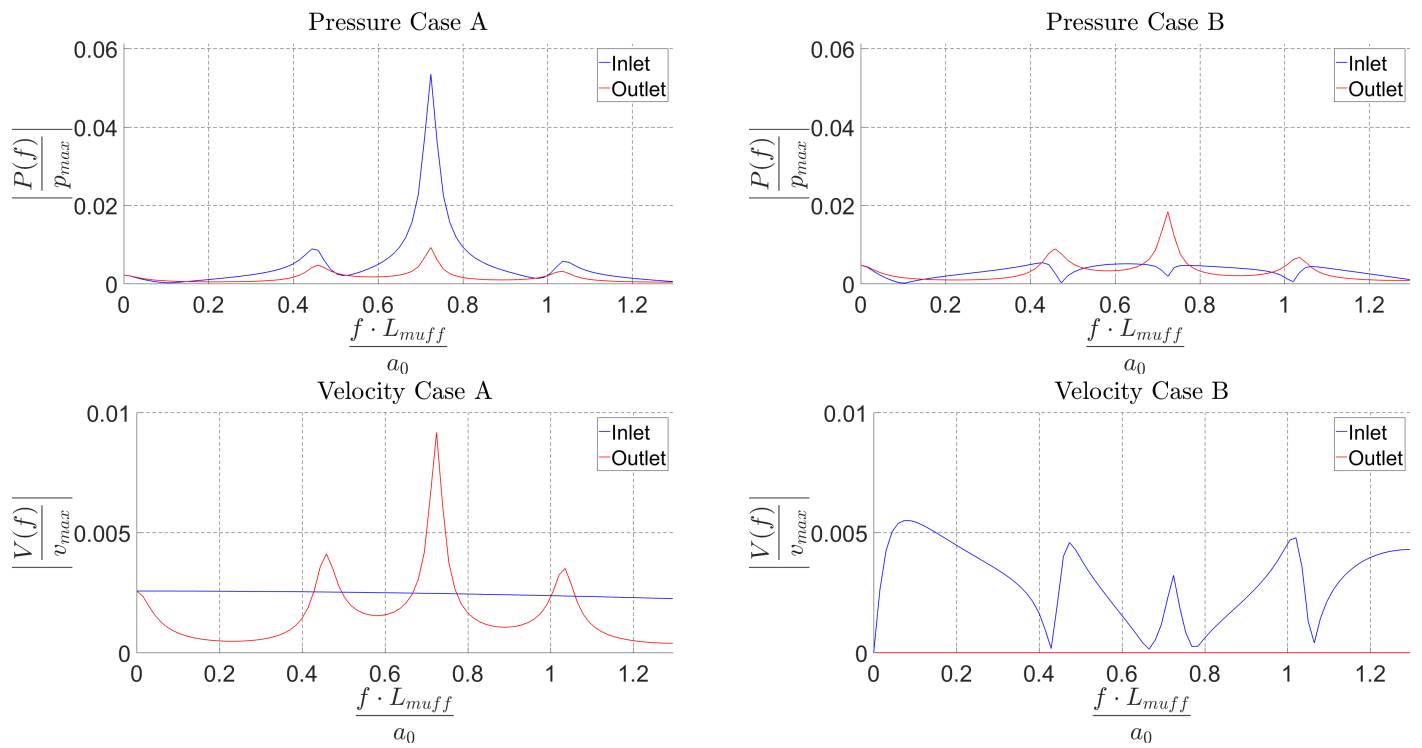

Figure 7: Frequency response of pressure and velocity at domain boundaries

It should be noticed that, as expected, the frequency response of the velocity at the inlet for case A is the same as that shown in Fig. 2 but it differs for Case B. The reason of this discrepancy is that, for Case B, the inlet boundary condition is assumed to be anechoic, so that the velocity components must be calculated and, for $t>T_{\text {impulse }}$, Equation (17) is not valid anymore.

\subsection{FEM frequency domain results}

Helmholtz equation is solved by means of FEM in the frequency domain. That leads to a significant decrease of the computational cost, compared with the CFD time domain method.

Fig. 8 shows the Sound Pressure Level $(S P L)$ in the domain, for different excitation frequencies. $S P L$ is defined in as:

$$
S P L(f)=20 \log _{10}\left(\frac{|P(f)|}{P_{r e f}}\right) \quad P_{r e f}=20 \mu \mathrm{Pa}
$$




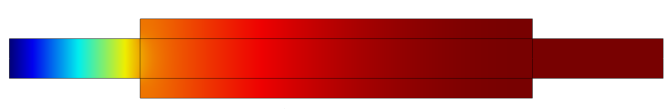

(a) $\frac{f L_{m u f f}}{a_{0}}=0.02$

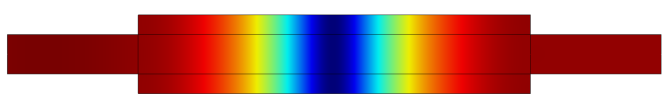

(c) $\frac{f L_{m u f f}}{a_{0}}=0.50$

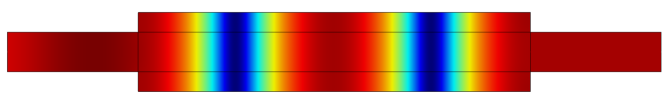

(e) $\frac{f L_{m u f f}}{a_{0}}=1.00$

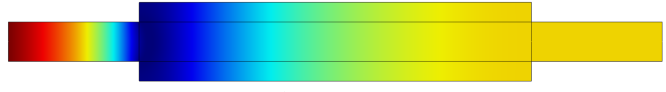

(b) $\frac{f L_{\text {muff }}}{a_{0}}=0.25$

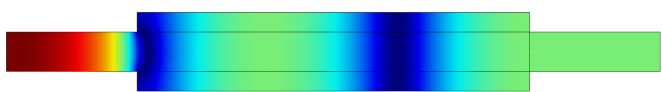

(d) $\frac{f L_{\text {muff }}}{a_{0}}=0.75$

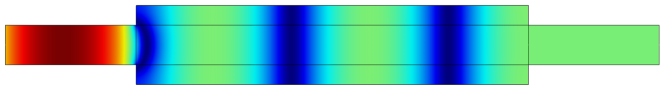

(f) $\frac{f L_{m u f f}}{a_{0}}=1.25$

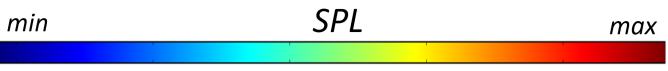

(g) Qualitative color map

Figure 8: SPL under different excitation frequencies

It can be noted from Fig. 8 that, for the frequencies $\frac{f L_{m u f f}}{a_{0}}=0.25, \frac{f L_{m u f f}}{a_{0}}=0.75$ and $\frac{f L_{m u f f}}{a_{0}}=1.25$ the $S P L$ at the outlet is much lower than the $S P L$ at the inlet. As it will be shown later, this corresponds to the frequencies of maximum attenuation. For the frequencies $\frac{f L_{m u f f}}{a_{0}}=0.50$ and $\frac{f L_{m u f f}}{a_{0}}=1.00$ the muffler enters into a resonance mode and no attenuation on the $S P L$ is found. For higher frequencies $3 \mathrm{D}$ effects are easier to be observed at the sudden section change zone.

\subsection{Characterisation of the rigid muffler features}

In this section the values of the transfer matrix will be obtained and compared using the methods presented in previous paragraphs.

The transfer matrix components are shown in Fig. 9
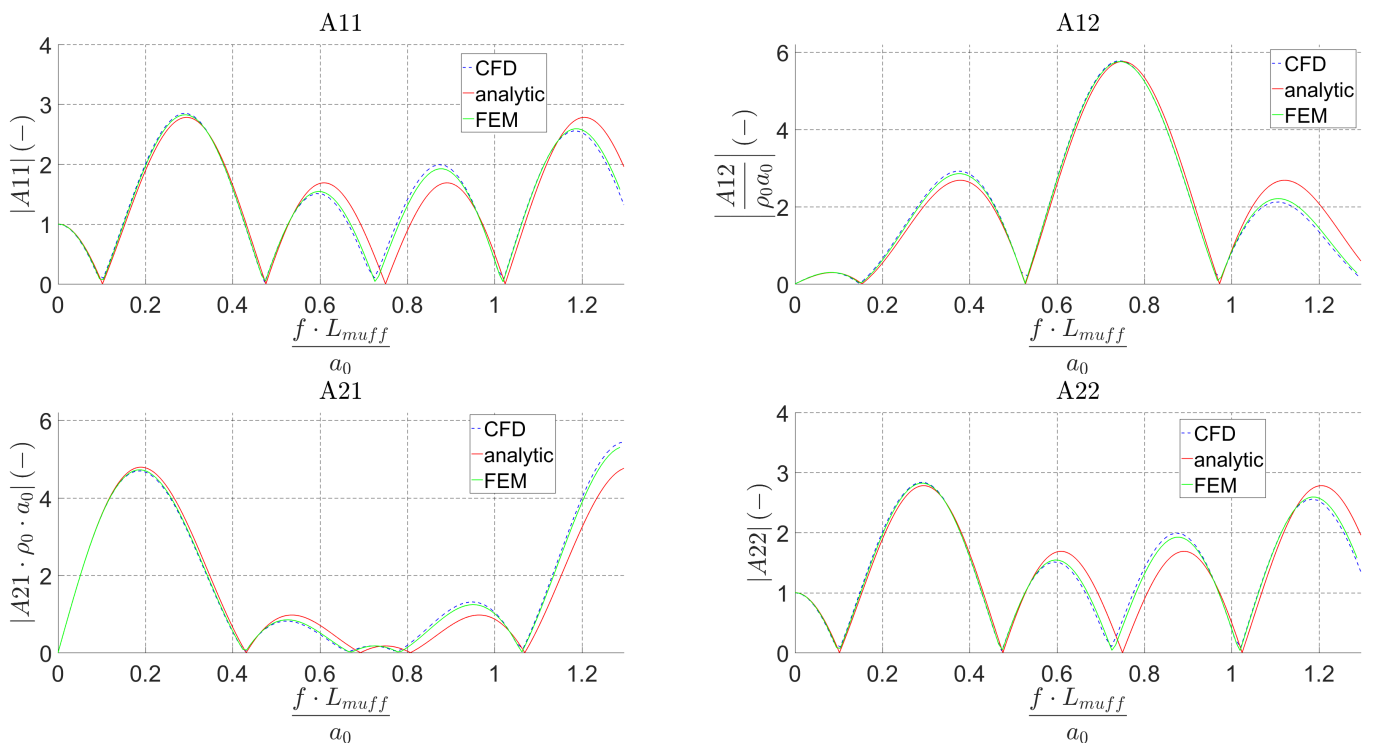

Figure 9: Transfer matrix coefficients 
All the previously explained methods coincide very well for low frequencies. Some discrepancies appear when quantifying values at higher frequencies. Nevertheless, this is mainly due to the onset of 3D effects, which are not taken into account by the analytical method. It will be later shown how those 3D issues barely affect the global characteristics of the system, such as Transmission Loss (and Insertion Loss, Velocity Ratio, etc.).

The Transmission Loss is shown, for each of the methods used, in Fig. 10. Notice how the analytical and the Finite Element method are perfectly coincident for the whole frequency range studied. For high frequencies the CFD study predicts a slightly higher value of the Transmission Loss. This could be primarily due to non-linear effects, which are taken into account in this method but are not in the analytical and FEM approximations.

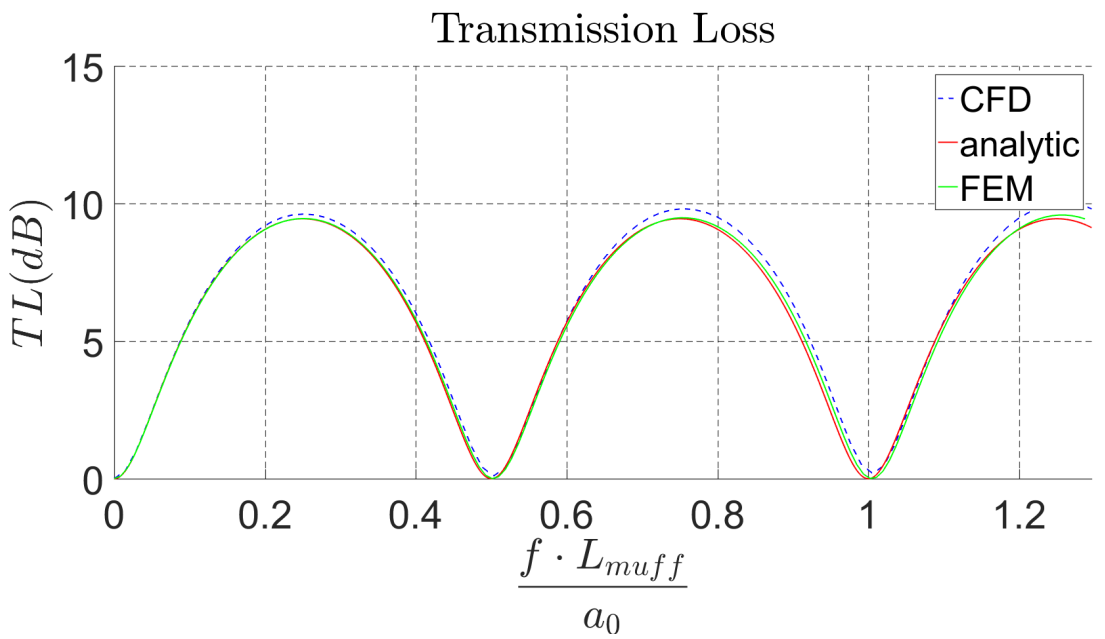

Figure 10: Transmission Loss as a function of frequency

These results show a nearly perfect agreement between predictions from a frequency domain FEM and from a time domain CFD calculation. This can be taken as a demonstration of the validity of both methods for these kind of problems. The following sections make use of these validated methods for the prediction of the particular features associated with a flexible expansion chamber.

\section{Results for the flexible expansion chamber}

\subsection{Flexible wall eigenfrequencies}

When analyzing the interaction between the acoustic field and the surrounding flexible wall, the excitation of the structural modes is of primal interest. It is thus necessary to perform an uncoupled analysis of those values.

Table 2 shows the first six eigenfrequencies of the quarter pipe, for the case of wall thickness $\delta=0.002 \mathrm{~m}$, wall density $\rho_{\text {wall }}=1000 \mathrm{~kg} \mathrm{~m}^{-3}$ and Poisson ratio $v=0.33$, and for various values of the Young modulus. These frequencies were calculated using the commercial software COMSOL Multiphysics for the isolated structure. As will be shown later apparition of axisymmetric modal shapes occur for higher values of frequency.

Fig. 11 shows the structural modal shape corresponding to each eigenfrequency calculated using COMSOL. Red colors correspond to maximum absolute values of the displacement while blue colors correspond to duct nodal lines. 
Table 2: Quarter pipe eigenfrequencies

\begin{tabular}{|c|c|c|c|c|}
\hline & \multicolumn{4}{|c|}{$f L_{\text {muff }} / a_{0}$} \\
\hline Mode & $E=0.1 \mathrm{GPa}$ & $E=0.5 \mathrm{GPa}$ & $E=1 \mathrm{GPa}$ & $E=30 \mathrm{GPa}$ \\
\hline 1 & 0.103 & 0.230 & 0.326 & 1.784 \\
\hline 2 & 0.178 & 0.398 & 0.563 & 3.084 \\
\hline 3 & 0.286 & 0.640 & 0.905 & 4.956 \\
\hline 4 & 0.409 & 0.913 & 1.292 & 7.076 \\
\hline 5 & 0.426 & 0.952 & 1.347 & 7.376 \\
\hline 6 & 0.436 & 0.976 & 1.380 & 7.558 \\
\hline
\end{tabular}

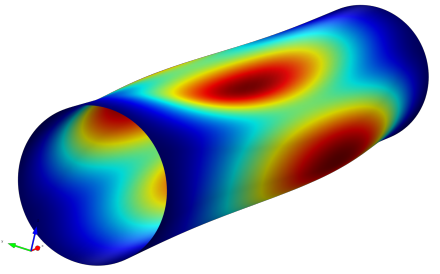

(a) Modal shape 1

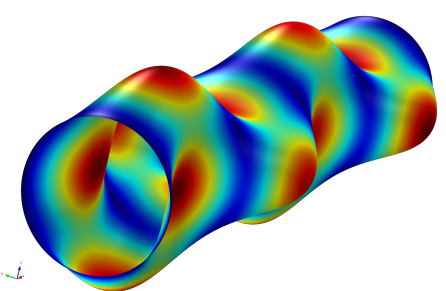

(d) Modal shape 4

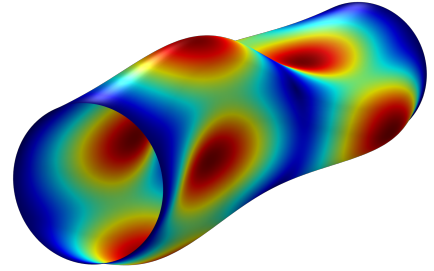

(b) Modal shape 2

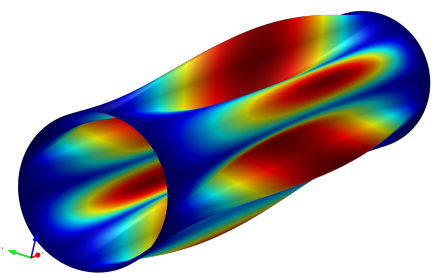

(e) Modal shape 5

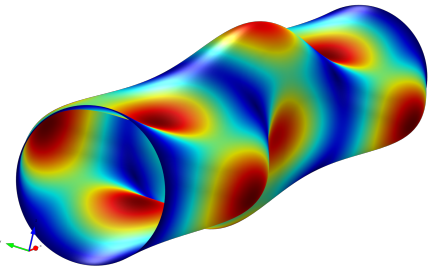

(c) Modal shape 3

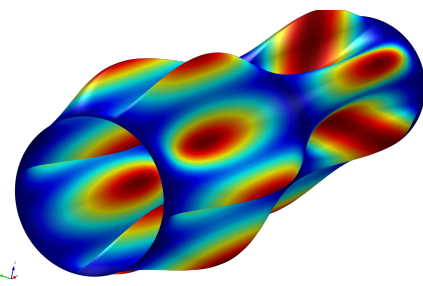

(f) Modal shape 6

Figure 11: Structural mode shapes

\subsection{CFD time domain results}

As the inlet tube is perfectly rigid, the maximum value of velocity and pressure during the pulse injection is exactly the same as in section 5. The inclusion of the FSI interaction leads to a significant increment of the computational cost. Thus, as the Transmission Loss is the most significant parameter when evaluating the performance of an acoustic element, only Case A was considered in this section. For the present case all the simulations were performed until a final time of $\frac{t a_{0}}{L_{\text {muff }}}=100$.

Fig. 12 and Fig. 13 show the time evolution of the pressure field for different time steps for case A (anechoic outlet), assuming the expansion chamber as flexible.

For illustration purposes, a parametric study of the influence of the Young modulus was performed. Fig. 12 shows the results for the case with $E=30 \mathrm{GPa}$ (pure tin) and Fig. 13 shows results for the case with $E=1 \mathrm{GPa}$ (polypropylene). The deformation field of the thin wall is also shown. Notice that the color-scale is shown in a qualitative manner in order to obtain interpretable representation.

- Fig. 12-13 a: The whole velocity-pressure pulse has been introduced into the domain. Cause the pulse has not arrived to the expansion zone, reflections or deformations do not appear yet.

- Fig. 12-13 b: The pulse reaches the inlet of the expansion chamber. The pressure is affected exactly by the same phenomena which were explained in section 5. Now, an axisymmetric deformation field can be observed both in the $E=30 \mathrm{GPa}$ and $E=1 \mathrm{GPa}$ being higher in the second case, as expected. 
- Fig. 12-13 c: The reflected pulse reaches the inlet. The boundary condition $v(t)=0$ behaves as a rigid wall, so it is reflected again towards the expansion chamber again. In the case $E=30 \mathrm{GPa}$ the deformation-pulse propagates together with the pressure pulse in the expansion chamber. In the case $E=1 \mathrm{GPa}$ it can be noticed how the low material stiffness leads to an additional wave.

- Fig. 12-13 d: The primary pulse reaches the outlet of the expansion chamber, and here a new partial reflection and transmission are found. The deformation pulse is also reflected. Note the high qualitative difference between the $E=30 \mathrm{GPa}$ and $E=1 \mathrm{GPa}$. In the second case it can be seen that the number of axisymmetric waves appearing is significantly higher.

- Fig. 12-13 e: The primary pulse reaches the outlet domain section. Due to the non-reflective boundary condition the outlet behaves as an anechoic termination. The pressure and deformation pulses continue travelling inside the expansion chamber, mutually interacting.

- Fig. 12-13 f: The primary pulse reaches again the inlet of the expansion chamber. Some part of the pulse is reflected towards the outlet, and some other passes through to the inlet of the domain.

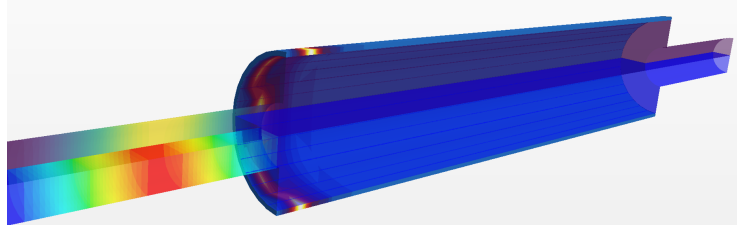

(a) $\frac{t \cdot a_{0}}{L_{m u f f}}=0.34$

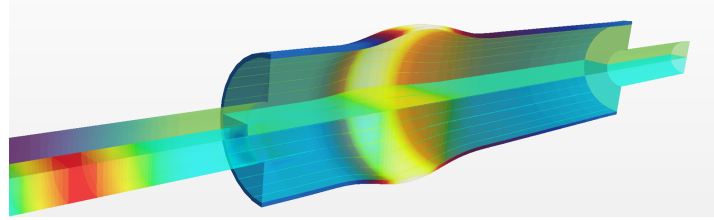

(c) $\frac{t \cdot a_{0}}{L_{m u f f}}=0.84$

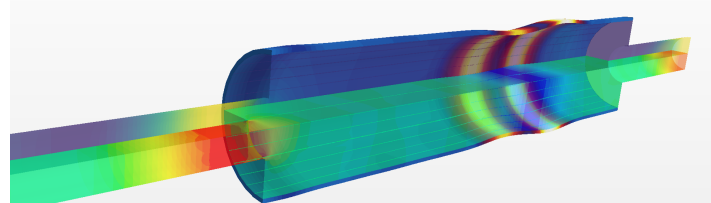

(e) $\frac{t \cdot a_{0}}{L_{m u f f}}=1.85$

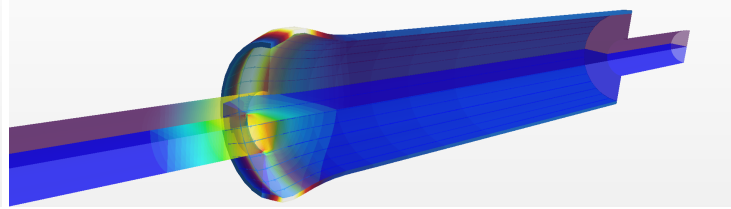

(b) $\frac{t \cdot a_{0}}{L_{m u f f}}=0.51$

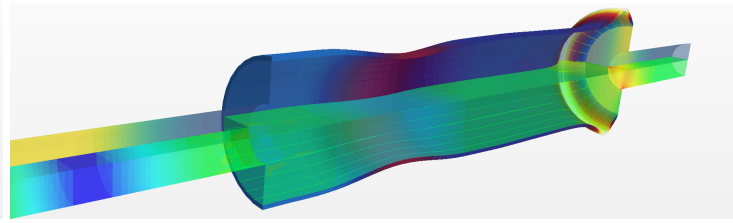

(d) $\frac{t \cdot a_{0}}{L_{m u f f}}=1.50$

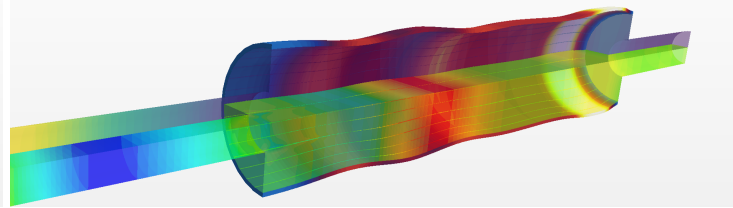

(f) $\frac{t \cdot a_{0}}{L_{m u f f}}=2.89$

\begin{tabular}{ccc}
\hline 0 & Displacement magnitude & $\max$ \\
\hline $\min$ & Axial velocity & $\max$ \\
\hline $\min$ & Pressure & $\max$
\end{tabular}

(g) Qualitative color map

Figure 12: Time evolution of the pressure and velocity pulse for different time instants and $E=30 \mathrm{GPa}$ 


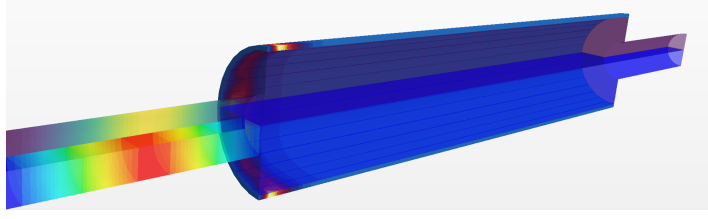

(a) $\frac{t \cdot a_{0}}{L_{m u f f}}=0.34$

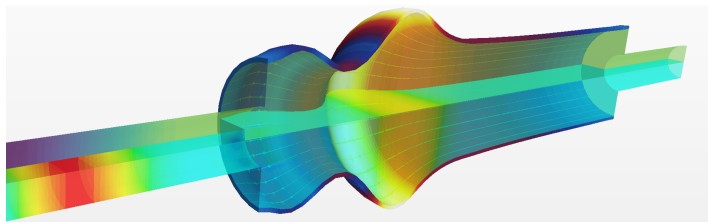

(c) $\frac{t \cdot a_{0}}{L_{m u f f}}=0.84$

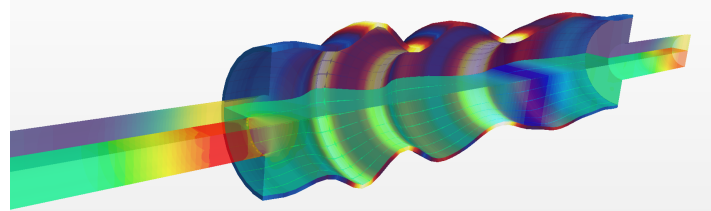

(e) $\frac{t \cdot a_{0}}{L_{m u f f}}=1.85$

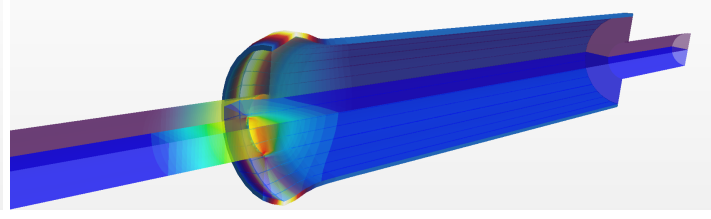

(b) $\frac{t \cdot a_{0}}{L_{m u f f}}=0.51$

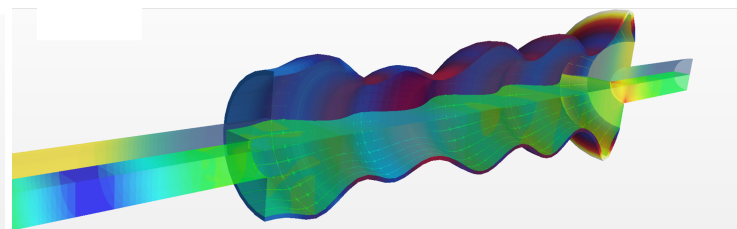

(d) $\frac{t \cdot a_{0}}{L_{m u f f}}=1.50$

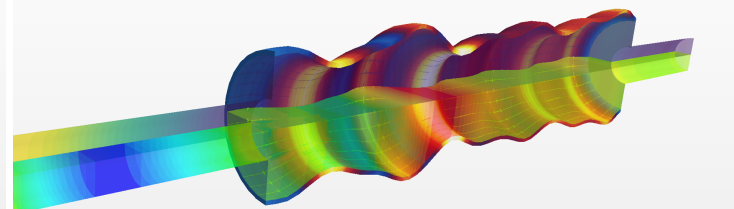

(f) $\frac{t \cdot a_{0}}{L_{m u f f}}=2.89$

\begin{tabular}{ccc}
\hline 0 & Displacement magnitude & $\max$ \\
\hline $\min$ & Axial velocity & $\max$ \\
\hline $\min$ & Pressure & $\max$
\end{tabular}

(g) Qualitative color map

Figure 13: Time evolution of the pressure and velocity pulse for different time instants and $E=1 \mathrm{GPa}$

Another important result, where differences between the rigid and the flexible cases can be found, is the time response of pressure and velocity at the inlet and the outlet. This time response is shown in Fig. 14 and, as it can be observed, no differences can be appreciated between the rigid case and the flexible case with $E=30 \mathrm{GPa}$. For cases of Young modulus $E<1 \mathrm{GPa}$ the time response shows significant deviations from the rigid case, specially after some reflections. It can be seen how, as a general trend, pressure peaks of reflections are lower as the value of rigidity decreases. 

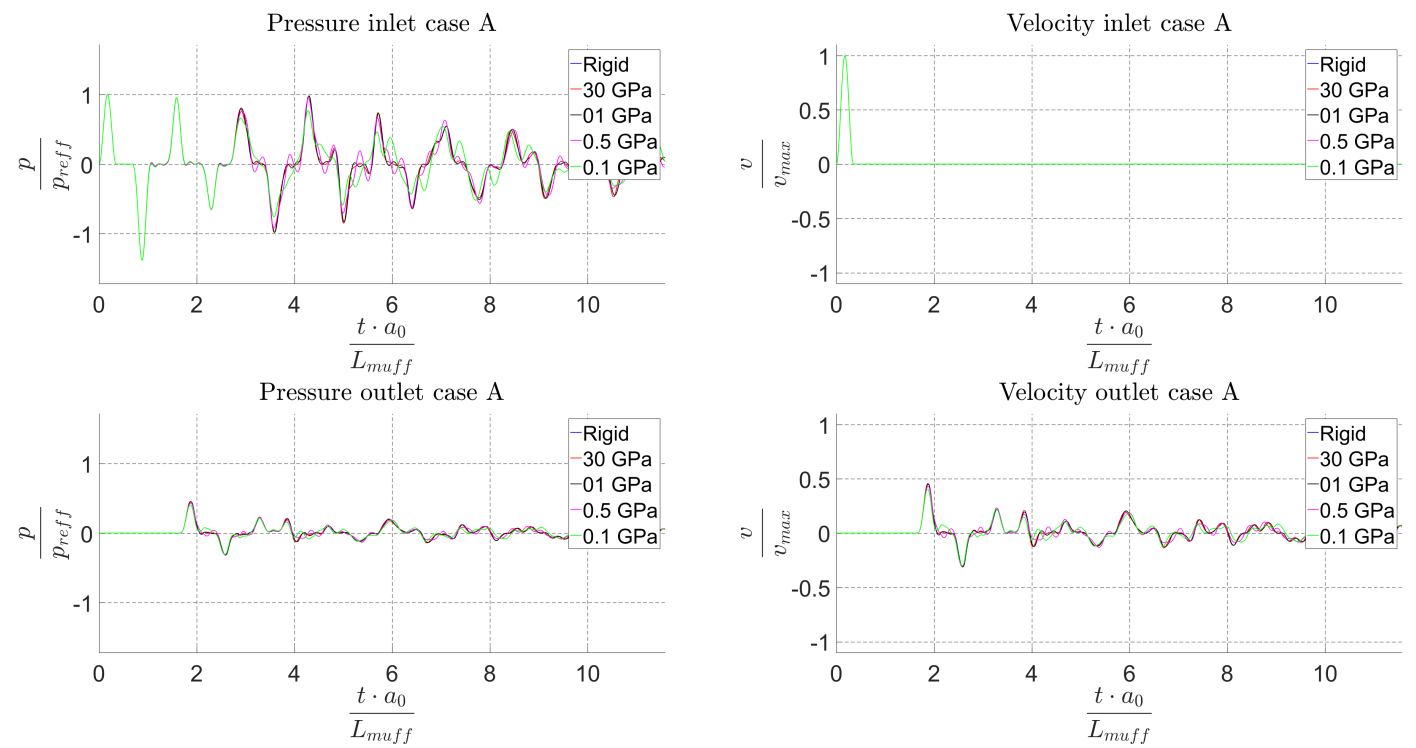

Figure 14: Time evolution of the pressure and velocity at domain boundaries for Case A. Different material rigidity

Fig. 15 shows the time evolution of deformation at a point located in the mid section of the expansion chamber, at a location $\left(L_{m u f f} / 2, R\right)$ for different wall rigidities. High differences in the displacement can be observed for the different materials. Fig. 15 also shows that the natural modes of the structure remain unattenuated. This is due to absence of damping in the model.
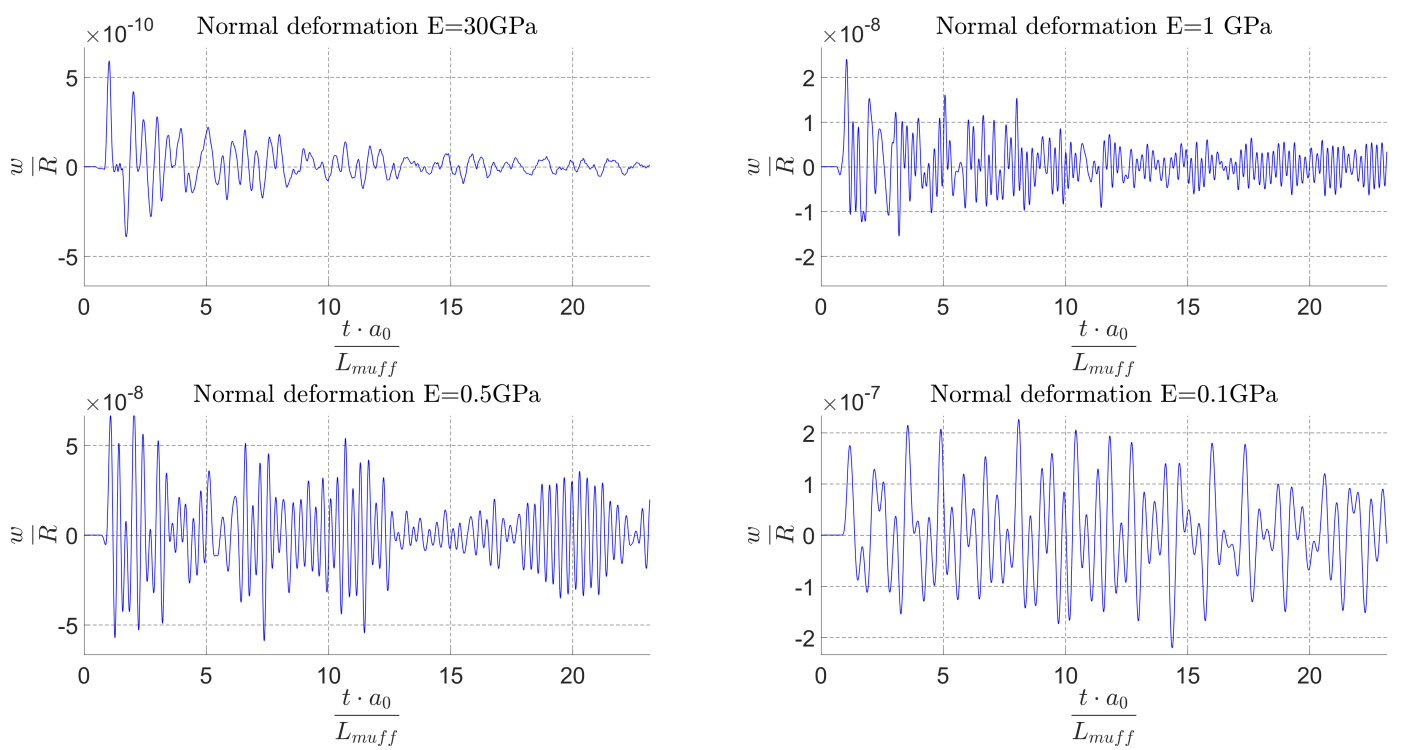

Figure 15: Time evolution of the wall displacement for Case A. Different material rigidity

Fig. 16 shows the frequency content of this radial displacement history. It can be observed that the frequency response is identical (except, obviously, in the absolute value) for the cases with $E=30 \mathrm{GPa}, E=1 \mathrm{GPa}$ and $E=0.5$ GPa. For these values of rigidity, two well-differentiated peaks are found at $\frac{f L_{\text {muffler }}}{a_{0}}=0.75$ and $\frac{f L_{\text {muffler }}}{a_{0}}=1.00$, which correspond with the chamber resonance frequencies. However, for the case with $E=0.1 \mathrm{GPa}$ a completely different 
response is obtained. A new, more important peak, appears at $\frac{f L_{\text {muffler }}}{a_{0}}=0.87$, which corresponds to the excitation of the first axisymmetric natural mode of the structure.
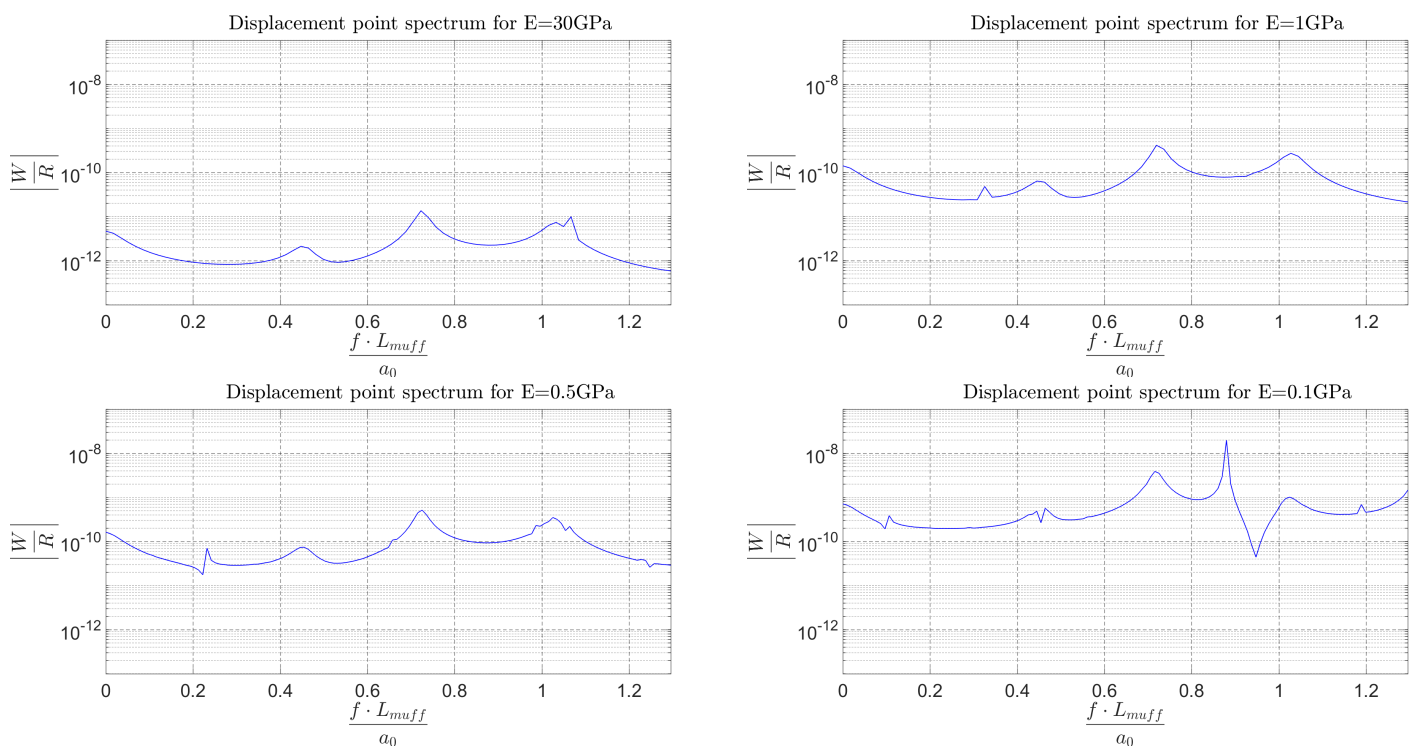

Figure 16: Frequency response of the radial wall displacement for Case A. Different material rigidity

\subsection{FEM frequency domain results and characterization of the flexible muffler}

Fig. 17 shows the Transmission Loss, calculated using Equation (21), for the flexible case with low Young modulus, $E=0.1 \mathrm{GPa}$. The two traces correspond to a calculation using time-domain CFD and a calculation using frequency-domain FEM.

Fig. 17 allows to demonstrate that, as well as for the rigid case (see Fig. 10), frequency domain FEM and time domain CFD provide similar results, with little discrepancies between both methods.

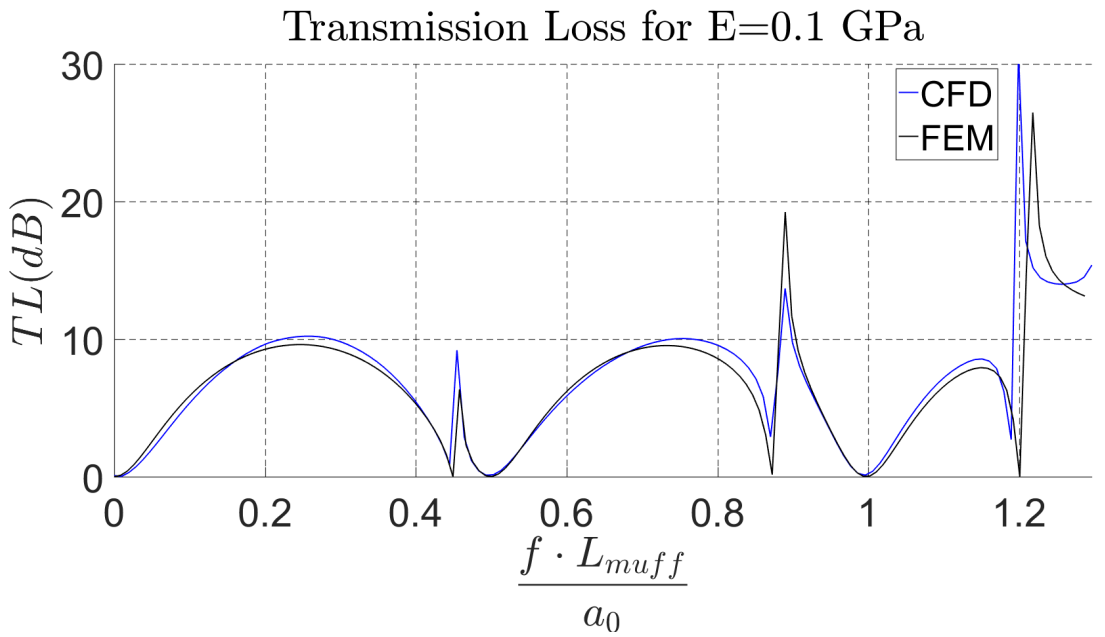

Figure 17: Transmission Loss as a function of frequency for $E=0.1 \mathrm{GPa}$. Comparison between time domain CFD model and frequency domain FEM model 
In order to examine the dependence of the acoustic response on rigidity, Fig. 18 shows the evolution of the Transmission Loss response as the Young modulus is decreased. In order to improve the interpretation of the results, they all are compared with the perfectly rigid result.

It can be observed that, for the two first cases $(E=1 \mathrm{GPa}$ and $E=0.5 \mathrm{GPa})$ non-noticeable differences are encountered between the rigid and the flexible cases. Nevertheless, for $E \leq 0.2 \mathrm{GPa}\left(D^{*} \leq 0.04\right)$ important differences appear. This coincides with the excitation of the first and second axisymmetric modes of the structure (see the location of these modes in Table 3). Figure 19 shows the modal shape corresponding to the first four eigenfrequencies.

Under these circumstances it can be found that, just before the axisymmetric mode is excited, the fluid-structure coupling leads to an important change in behavior. Around this frequency a high attenuation point is found immediately followed or preceded by a sharp pass band of abrupt attenuation drop. This sharp behavior is due to the strong fluid-structure coupling derived from the excitation of the natural axisymmetric modes of the structure. Similar behavior was found in the transfer function studied by Herrmann et al. [33] for the case of thin hydraulic pipes.
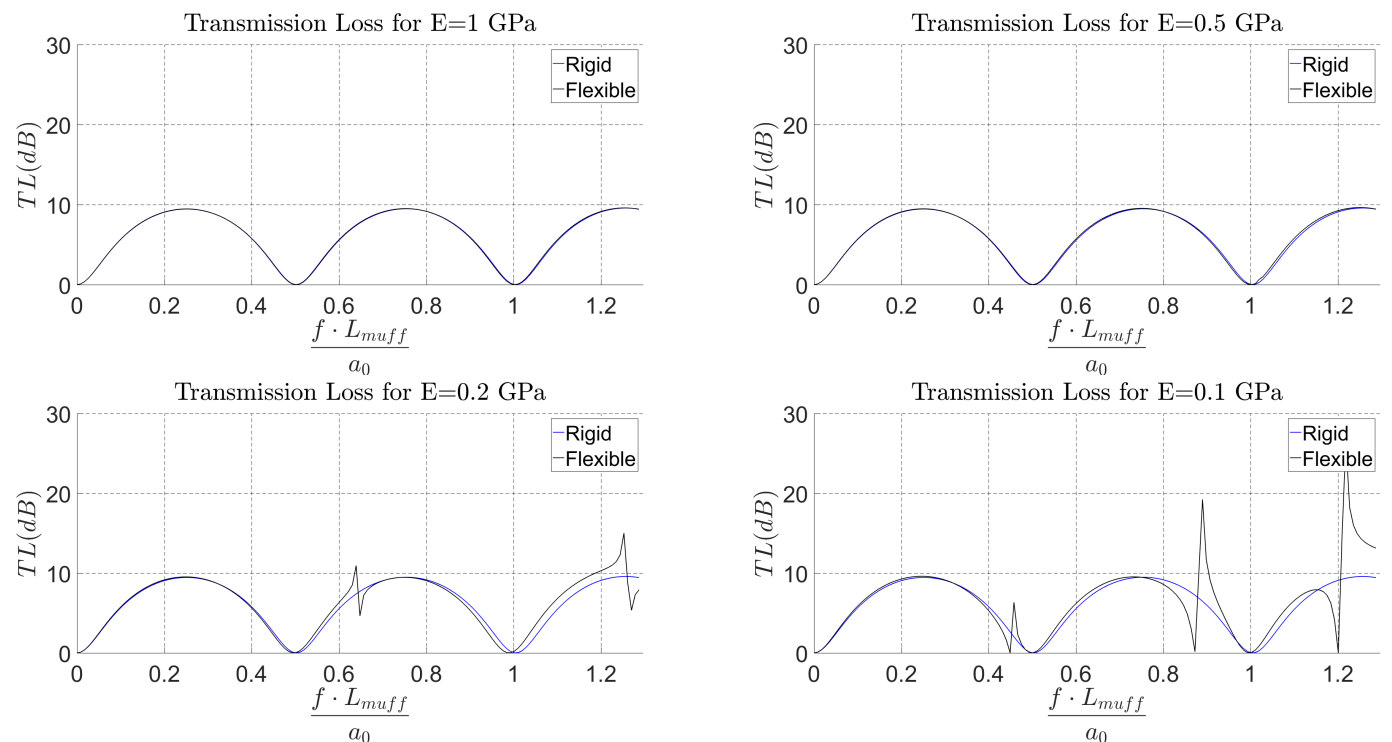

Figure 18: Transmission Loss as a function of stiffness for mass parameter $m_{l}^{*}=57$

Table 3: Axisymmetric modes for different non-dimensional stiffness parameter, $D^{*}$

\begin{tabular}{|c|c|c|c|c|}
\hline & \multicolumn{4}{|c|}{$f L_{\text {muff }} / a_{0}$} \\
\hline Mode & $D^{*}=0.02$ & $D^{*}=0.04$ & $D^{*}=0.10$ & $D^{*}=0.20$ \\
\hline 1 & 0.45 & 0.64 & 1.01 & 1.43 \\
\hline 2 & 0.89 & 1.25 & 1.98 & 2.80 \\
\hline 3 & 1.21 & 1.71 & 2.71 & 3.83 \\
\hline 4 & 1.33 & 1.89 & 2.98 & 4.22 \\
\hline 5 & 1.37 & 1.94 & 3.06 & 4.33 \\
\hline 6 & 1.39 & 1.96 & 3.10 & 4.38 \\
\hline
\end{tabular}




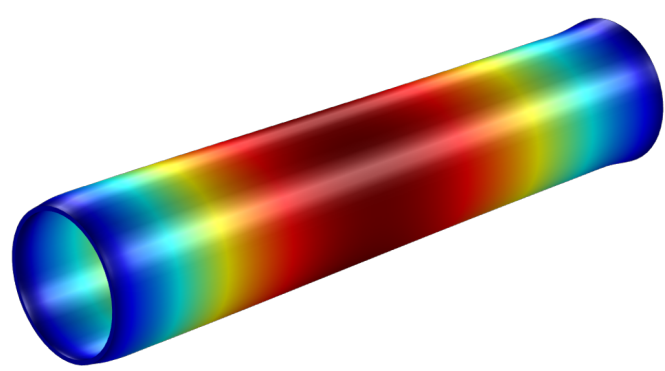

(a) Axisymmetric modal shape 1

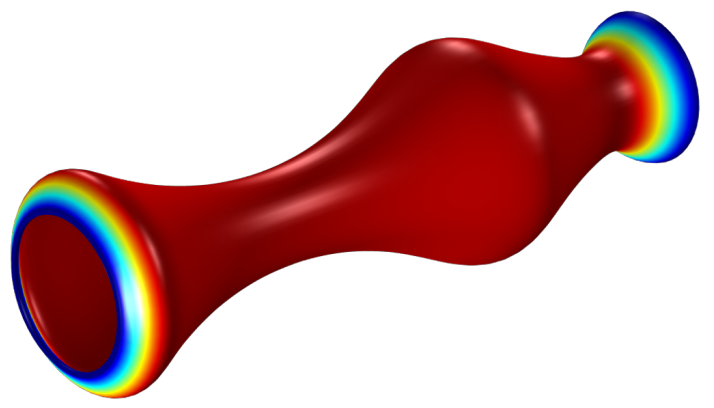

(c) Axisymmetric modal shape 3

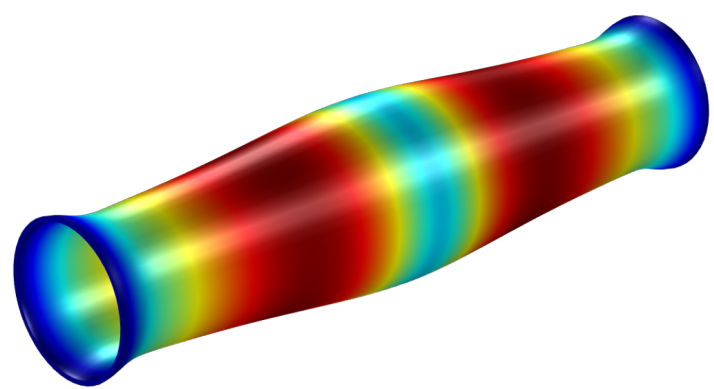

(b) Axisymmetric modal shape 2

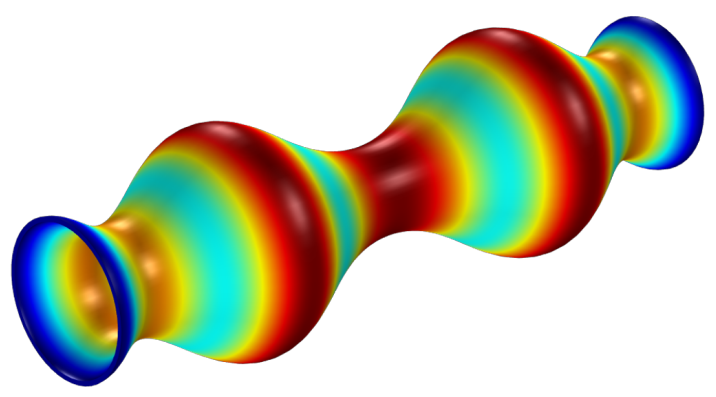

(d) Axisymmetric modal shape 4

Figure 19: Axisymmetric structural mode shapes

Fig. 20 and Fig. 21 show the frequency response field of Sound Pressure Level and displacement, respectively, for the case A with $E=0.1 \mathrm{GPa}$. It can be observed that, as previously predicted, the cases of minimum acoustic losses $S L P_{\text {out }} \approx S P L_{\text {in }}$ correspond with the excitation of a structural axisymmetric mode.

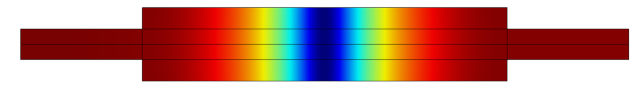

(a) $\frac{f L_{\text {muff }}}{a_{0}}=0.45$

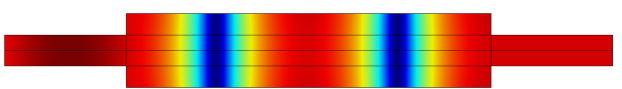

(c) $\frac{f L_{m u f f}}{a_{0}}=0.87$

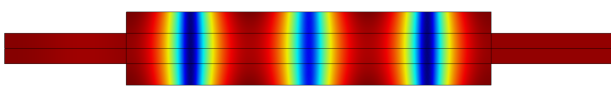

(e) $\frac{f L_{\text {muff }}}{a_{0}}=1.20$

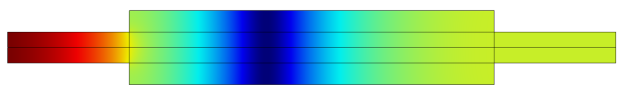

(b) $\frac{f L_{m u f f}}{a_{0}}=0.46$

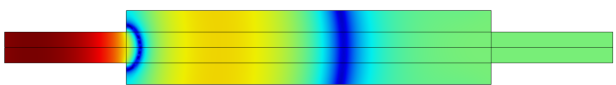

(d) $\frac{f L_{m u f f}}{a_{0}}=0.89$

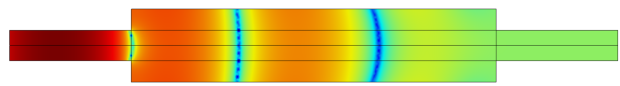

(f) $\frac{f L_{m u f f}}{a_{0}}=1.22$

$\min \quad S P L \quad \max$

(g) Qualitative color map

Figure 20: SPL under different excitation frequencies for the flexible case 


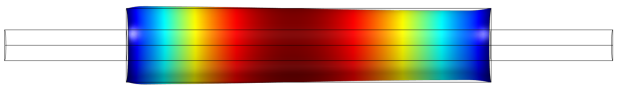

(a) $\frac{f L_{m u f f}}{a_{0}}=0.45$

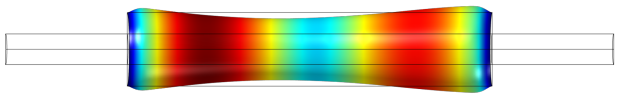

(c) $\frac{f L_{m u f f}}{a_{0}}=0.87$

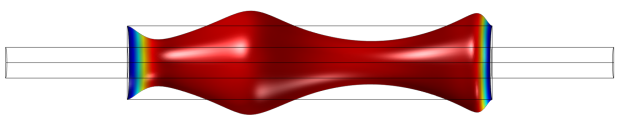

(e) $\frac{f L_{m u f f}}{a_{0}}=1.20$

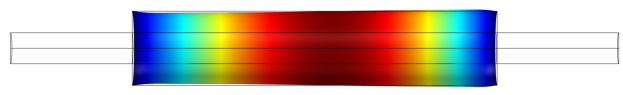

(b) $\frac{f L_{m u f f}}{a_{0}}=0.46$

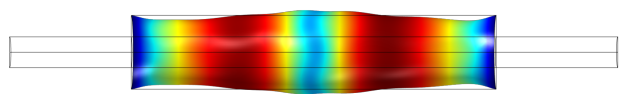

(d) $\frac{f L_{m u f f}}{a_{0}}=0.89$

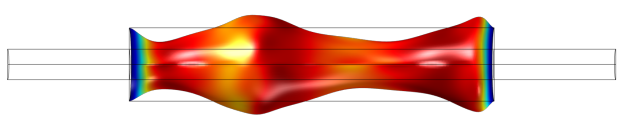

(f) $\frac{f L_{m u f f}}{a_{0}}=1.22$

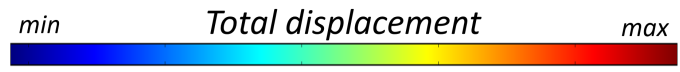

(g) Qualitative color map

Figure 21: Displacement under different excitation frequencies for the flexible case

Fig. 22 shows the evolution of the strain and kinetic energy stored in the solid. Notice how the maximum peak values of both energies correspond to the excitation of the axisymmetric natural modes.

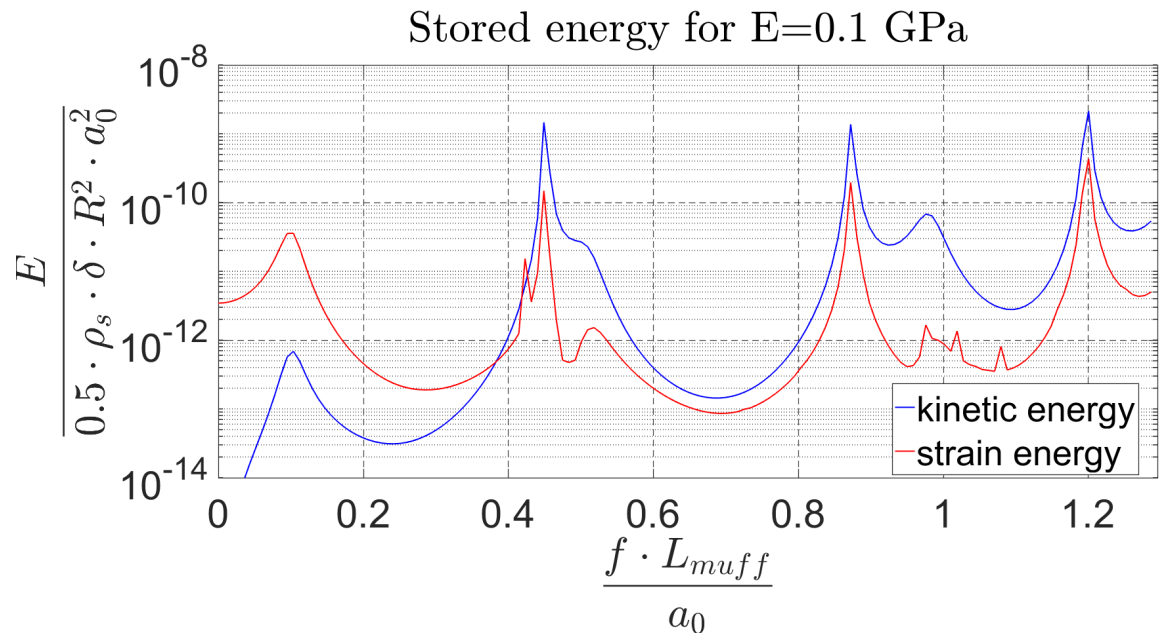

Figure 22: Stored energy as a function of the frequency for $E=0.1 \mathrm{GPa}$

\section{Conclusions}

A methodology based on CFD-FEM method has been presented for the Transmission Loss calculation of vibroacoustic domains both in time and frequency domain.

A review of the main different techniques used for the characterization of vibro-acoustic problems has been performed.

To fine tune the methodology under consideration, different simulations were performed using both CFD and FEM in order to predict the acoustic performance of a perfectly rigid expansion chamber. Results show very good agreement between CFD, FEM and analytic cases. 
Similar methods were later used for the case of an expansion chamber with flexible walls. Again, the presented models show good agreement for the studied frequency range.

Nevertheless, it was observed that computational requirements of the time-domain CFD method are various order of magnitude higher than the requirements for the same geometry using a frequency-domain FEM approach.

General trends of the vibroacoustic response were calculated for a rigid and a flexible expansion chamber. Results show that, for the current geometry, the influence of rigidity for a heavy structure is important only for very low Young modulus. The effect of the structural vibration becomes important when excitation of the first and second axisymmetric natural modes of the structure takes place.

It was demonstrated that, when the inner flow excites the structure at the frequency of its natural modes, the problem suddenly becomes strongly coupled and the influence of the flexible walls should be taken into account.

Finally, it was illustrated that, for a geometry of the shapes and sizes like the simplified cylindrical expansion chamber presented during the current work, the mentioned complete coupling only appears for very low values of the Young modulus at the frequencies of interests. As this phenomena is mainly due to the excitation of the natural modes of the structure, mufflers with higher modal density should experience this phenomena even for higher values of the Young Modulus.

\section{Acknowledgments}

This work has been partially supported by the Spanish Ministerio de Economía y Competitividad through Grant No. DPI2015-70464-R and Generalitat Valenciana by means of project Prometeo/2016/007.

\section{References}

[1] W. H. O. for the European Union, Night Noise Guidelines for Europe, Tech. Rep., 2010.

[2] E. Union, On the sound level of motor vehicles and of replacement silencing systems. Regulation (EU) No 540/2014 of the european parliament and of the council of 16 April 2014, Tech. Rep., 2014.

[3] C. H. Hansen, Understanding active noise cancellation, Spon Press, 2003.

[4] Y. L. A. Linus, Y. K. Koh, H. P. Lee, The performance of active noise-cancelling headphones in different noise environments, Applied Acoustics 122 (2017) 16-22.

[5] H. D. Hwang, K. Ege, Y. Gerges, J. L. Guyader, SmEdA vibro-acoustic modelling in the mid-frequency range including the effect of dissipative treatments, Journal of Sound and Vibration 393 (2017) 187-215.

[6] M. L. Munjal, Acoustics of Ducts and Mufflers - Second Edition, Wiley, 2014.

[7] B. Aydemir, A. Ebrin, Effect of Material Properties and Wall Thickness of Polymer Based Intake Manifold on the Engine Radiated Noise Levels, SAE International 2001-01 (2009) 1544.

[8] R. F. Nunes, C. F. Nogueira, M. A. Argentino, D. Hackenbroich, Developement of an Air Intake System Using Vibro-Acoustics Numerical Modeling, SAE International 2001-01 (2001) 1519.

[9] K. Hu, C. Lee, E. Homsi, D. Moenssen, Acoustic Modeling and Radiated Noise Prediction for Plastic Air-Intake Manifolds, SAE International 2003-01 (2003) 1448 .

[10] L. Huang, Modal analysis of a drumlike silencer, The Journal of the Acoustical Society of America 112 (2002) 2014-2015.

[11] H. K. Fan, R. C. Leung, G. C. Lam, Numerical analysis of aeroacoustic-structural interaction of a flexible panel in uniform duct flow, The Journal of the Acoustical Society of America 137 (2015) 3115-3126.

[12] J. B. Lawrie, Analytic mode-matching for acoustic scattering in three dimensional waveguides with flexible walls: Application to a triangular duct, Wave Motion 50 (2013) 542-557.

[13] S. Ramamoorthy, S. Grosh, J. M. Dodson, A theoretical study of structural acoustic silencers for hydraulic systems, The Journal of the Acoustical Society of America 111 (2012) 2097-2108

[14] S. H. Ko, Sound Wave Propagation In A Two-dimensional Flexible Duct In The Presence Of An Inviscid Flow, Journal of Sound and Vibration 175 (1994) 279-287.

[15] F. Gautier, J. Gilbert, J. P. Dalmont, R. P. Vila, Wave Propagation in a Fluid Filled Rubber Tube: Theoretical and Experimental Results for Korteweg's Wave, Acta Acustica united with Acustica 93 (2007) 333-344.

[16] B. Venkatesham, M. Tiwari, M. L. Munjal, Analytical prediction of break-out noise from a reactive rectangular plenum with four flexible walls, The Journal of the Acoustical Society of America 128 (2010) 1789-1799.

[17] G. Wang, W. L. Li, J. Du, W. Li, Prediction of break-out sound from a rectangular cavity via an elastically mounted panel, The Journal of the Acoustical Society of America 139 (2016) 684-692.

[18] M. L. Munjal, P. T. Thawani, Prediction of the vibro-acoustic transmission loss of planar hose-pipe systems, The Journal of the Acoustical Society of America 101 (1998) 2524-2535.

[19] A. Cummings, The attenuation of sound in unlined ducts with flexible walls, Journal of Sound and Vibration 174 (1994) $433-450$.

[20] Y. Bazilevs, K. Takizawa, T. E. Tezduyar, Computational Fluid-Structure Interaction. Methods and Applications, Wiley, 2013.

[21] O. C. Zienkiewicz, R. L. Taylor, D. Fox, The Finite Element Method for Solid and Structural Mechanics, Elsevier, 2014.

[22] F. Irgens, Continuum mechanics, Springer, 2008. 
[23] F. Anselmet, P. O. Mattei, Acoustics, Aeroacoustics and Vibrations, Wiley, 2016.

[24] Y. Inoue, Y. Gotoh, Formation of weak shock waves caused by a sphere pulsating at large amplitude and low frequency, Journal of Sound and Vibration 145 (1991) 269-280.

[25] M. J. Lighthill, Viscosity effect in sound waves of finite amplitude, Survey in Mechanics 250 (1956) 337-348.

[26] E. C. Romfo, M. D. Campos, L. F. M. Moura, Application of the Galerkin and Least-Squares Finite Element Methods in the solution of 3D Poisson and Helmholtz equations, Computers and Mathematics with Applications 62 (2011) 4288-4299.

[27] COMSOL Multiphysics 5.2 User's Guide, 2016

[28] A. Broatch, X. Margot, A. Gil, F. Denia, A CFD approach to the computation of the acoustic response of exhaust mufflers, Journal of Computational Acoustics 13 (2005) 301-316.

[29] G. Liu, X. Zhao, W. Zhang, S. Li, Study on plate silencer with general boundary conditions, Journal of Sound and Vibration 333 (2014) 4881-4896.

[30] R. Barbieri, N. Barbieri, Finite element acoustic simulation based shape optimization of a muffler, Applied Acoustics 67 (2006) $346-357$.

[31] STARCCM+ 12.02.010 User's Guide, 2016.

[32] F. J. Fuenmayor, F. D. Denia, J. Albelda, E. Giner, H-adaptive refinement strategy for acoustic problems with a set of natural frequencies, Journal of Sound and Vibration 255 (2002) 457-479.

[33] J. Herrmann, J. Koreck, M. Matthias, L. Gaul, O. Estorff, Frequency-dependent damping model for the hydroacoustic finite element analysis of fluid-filled pipes with diameter changes, Mechanical Systems and Signal Processing 25 (2011) 981-990.

\section{Nomenclature}

$\rho \quad$ Density

$\vec{w} \quad$ Solid displacement

$\sigma \quad$ Solid Stress tensor

$\vec{b} \quad$ Solid volume forces

$\vec{f}_{m} \quad$ Fluid volume forces

$Q \quad$ Fluid volume heat generation

$\phi_{v} \quad$ Viscous dissipation

$\varepsilon \quad$ Solid strain

$\lambda \quad$ 1st Lamé parameter

$\mu \quad$ 2nd Lamé parameter or viscosity (according to context)

$E \quad$ Young modulus

$c_{p} \quad$ Specific heat

$v \quad$ Poisson ratio

$u \quad$ Fluid velocity

$\tau \quad$ Fluid stress tensor

$p \quad$ Pressure

${ }_{453} \quad T \quad$ Temperature

$h \quad$ Enthalpy

$c_{p} \quad$ Specific heat

$\vec{n} \quad$ Surface normal vector

$t \quad$ Time

a Sound speed

$k \quad$ Thermal conductivity

$k_{0} \quad$ Wave number

$j \quad$ Imaginary unit

$R \quad$ Radius or gas constant (according to context)

$\delta \quad$ Thickness or Dirac Delta (according to context)

[A] Transfer matrix

$T L$ Transmission Loss

$V R \quad$ Velocity ratio

$f \quad$ Frequency

$h \quad$ Enthalpy

$T_{S} \quad$ Sampling time

[M] Mass matrix 


$\begin{array}{ll}{[C]} & \text { Damping matrix } \\ {[K]} & \text { Stiffness matrix } \\ \omega & \text { Angular frequency } \\ L_{m u f f} & \text { Expansion chamber length } \\ m^{*} & \text { Mass ratio } \\ D^{*} & \text { Stiffness parameter } \\ v & \text { Velocity at the boundary conditions } \\ \text { Subscripts and superscripts } & \\ 0 & \text { Unperturbed case } \\ \text { ref } & \text { Reference data } \\ \text { in } & \text { Inlet } \\ \text { out } & \text { Outlet } \\ * & \text { Non dimensional variable }\end{array}$

\section{List of Tables}

Table $1 \quad$ Geometry dimensions $\ldots \ldots \ldots \ldots \ldots \ldots \ldots \ldots$

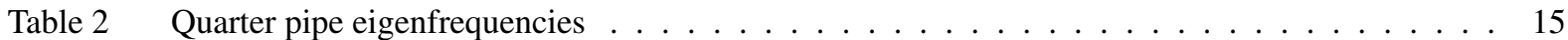

Table 3 Axisymmetric modes for different non-dimensional stiffness parameter, $D^{*} \ldots \ldots \ldots$

\section{List of Figures}

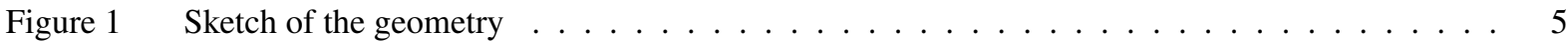

Figure 2 Time and frequency response of the inlet velocity profile . . . . . . . . . . . . . 6

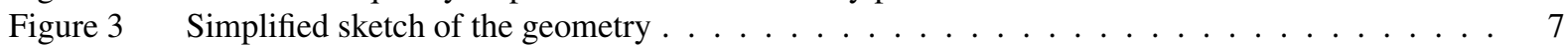

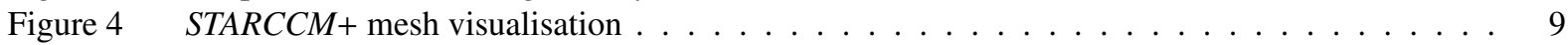

Figure $5 \quad$ COMSOL Fluid domain mesh . . . . . . . . . . . . . . . . . . . . . 10

Figure 6 Time evolution of the pressure and velocity at domain boundaries . . . . . . . . . . . 11

Figure 7 Frequency response of pressure and velocity at domain boundaries . . . . . . . . . . . . 12

Figure 8 SPL under different excitation frequencies . . . . . . . . . . . . . . . . 13

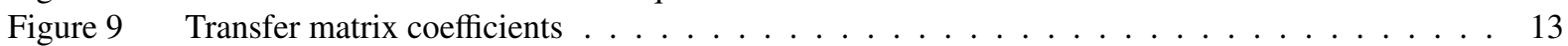

Figure 10 Transmission Loss as a function of frequency . . . . . . . . . . . . . . . . . . . . . . . 14

Figure 11 Structural mode shapes . . . . . . . . . . . . . . . . . . . . 15

Figure 12 Time evolution of the pressure and velocity pulse for different time instants and $E=30 \mathrm{GPa} \quad . \quad 16$

Figure 13 Time evolution of the pressure and velocity pulse for different time instants and $E=1 \mathrm{GPa} \quad$. 17

Figure 14 Time evolution of the pressure and velocity at domain boundaries for Case A. Different material

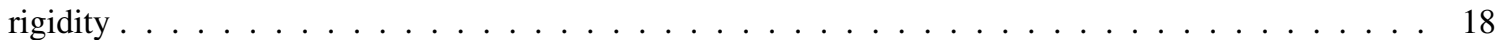

Figure 15 Time evolution of the wall displacement for Case A. Different material rigidity . . . . . . . . . 18

Figure 16 Frequency response of the radial wall displacement for Case A. Different material rigidity . . . 19

Figure 17 Transmission Loss as a function of frequency for $E=0.1 \mathrm{GPa}$. Comparison between time

domain CFD model and frequency domain FEM model . . . . . . . . . . . . . . . . . . . 19

Figure 18 Transmission Loss as a function of stiffness for mass parameter $m_{l}^{*}=57 \ldots \ldots$. . . . . . 20

Figure 19 Axisymmetric structural mode shapes . . . . . . . . . . . . . . . . . . 21

Figure 20 SPL under different excitation frequencies for the flexible case . . . . . . . . . . . . 21

Figure 21 Displacement under different excitation frequencies for the flexible case . . . . . . . . . . . 22

Figure 22 Stored energy as a function of the frequency for $E=0.1 \mathrm{GPa} \ldots \ldots \ldots$. . . . . . . . 22 